\title{
Paclitaxel-resistant cancer cell-derived secretomes elicit ABCB1-associated docetaxel cross-resistance and escape from apoptosis through FOXO3a-driven glycolytic regulation
}

\author{
Mark Borris D Aldonza ${ }^{1,2,3}$, Ji-Young Hong ${ }^{1}$ and Sang Kook Lee ${ }^{1}$
}

Chemotherapy-induced cancer cell secretomes promote resistance due, in part, to a predominant glycolytic energy metabolism, which drives aggressive cancer cell proliferation. However, the characterization of these secretomes and the molecular events that associate them with acquired drug resistance remain poorly understood. In this study, we show that secretomes of cancer cells with high-level paclitaxel resistance stimulated cell proliferation and suppressed drug-induced apoptosis of drug-sensitive cells. We also found that drug (docetaxel)-stimulated induction of interferon- $\alpha$ (IFN- $\alpha$ ), IFN- $\lambda$ and tumor necrosis factor- $\alpha$ $(\mathrm{TNF}-\alpha)$ release in drug-sensitive cells was lowered by these secretomes. The promotion of cell proliferation by paclitaxelresistant (PacR) cancer cell secretomes was associated, in part, with an increase in S phase of the cell cycle and downregulation of the cell death pathway that supports escape from apoptosis. In addition, we also found that the regulation of targeted glycolysis in PacR cancer cells alters the effects of the secretomes on cell growth, apoptosis, ATP generation and acquired drug resistance. Further study revealed that the deletion of FOX03a transcription exacerbates glycolytic shift-induced apoptosis by rescuing TRAIL expression. By generating a docetaxel-cross-resistant PacR cancer cell line (PacR/DCT), we further clarified the role of FOXO3a in glycolysis-associated mediation of P-glycoprotein/ABCB1 hyperactivity that induces docetaxel cross-resistance. These findings suggest that suppression of the cellular energy supply by targeting glycolysis may inhibit the multiplicity of acquired chemotherapy resistance. Therefore, the therapeutic inhibition of FOXO3a might direct glycolysis to induce apoptosis and overcome multidrug resistance in cancer cells.

Experimental \& Molecular Medicine (2017) 49, e286; doi:10.1038/emm.2016.131; published online 20 January 2017

\section{INTRODUCTION}

Acquired chemoresistance induces aggressiveness and causes relapse in a variety of cancer types. This resistance limits the efficacy of targeted therapies after a majority of patients show disease stabilization, which hampers the success of clinical treatments and increases the risk of death. ${ }^{1-3}$ Paclitaxel is the main treatment, along with platinum therapy, for ovarian, lung, prostate and breast cancer. Paclitaxel interrupts the dynamic equilibrium of tubulins and stabilizes the microtubule structure. Although studies have uncovered the mechanisms of paclitaxel resistance (PacR) in several malignancies, $\mathrm{PaCR}$ remains a complex and unsolved issue in the clinical setting. Various mechanisms have been implicated in PacR, including the following: increased P-glycoprotein (P-gp), which is encoded by ABCB1 (MDR1); associated drug efflux; blocked death signals; changed microtubulin dynamics; and altered stress responses, such as the activation of DNA repair and detoxification signals. ${ }^{4-7}$ However, clinical agents that regulate these mechanisms, such as P-gp inhibitors, are often ineffective or toxic at the doses required to induce efficacy. ${ }^{8}$ Therefore, current options for overcoming PacR are limited, necessitating the identification of more selective cancer therapies.

Altered energy metabolism (Warburg effect) has been recognized as one of the hallmarks of cancer. It has been demonstrated that the metabolic properties of drug-resistant cancer cells are different from those of drug-sensitive cancer cells, the same is true for cancer cells versus normal cells. ${ }^{9}$ Dysregulated cellular metabolism has been linked to

${ }^{1}$ College of Pharmacy, Seoul National University, Seoul, Korea and ${ }^{2}$ Department of Biochemistry, College of Veterinary Medicine, Seoul National University, Seoul, Korea

${ }^{3}$ Current address: Department of Biological Sciences and Department of Chemical and Biomolecular Engineering, Korea Advanced Institute of Science and Technology (KAIST), Daejeon 34141, Republic of Korea.

Correspondence: Professor SK Lee, College of Pharmacy, Seoul National University, 1 Gwanak-ro, Gwanak-gu, Seoul 08826, Korea.

E-mail: sklee61@snu.ac.kr

Received 2 March 2016; revised 5 September 2016; accepted 7 September 2016 
development of drug-resistant phenotypes, increased autophagy levels and regulation of critical glycolysis-associated molecules. Targeting dysregulated glucose metabolism overcomes therapeutic resistance in a number of models involving different mechanisms. ${ }^{10}$ Targeting glycolysis has been assessed using different approaches, one of the most frequent techniques used is modifying the glucose content in cells. Under glucose deprivation (GD), cells conserve energy to ensure survival and other related functions. ${ }^{11}$ Prolonged GD induces cellular stress, which regulates glucose-regulated protein 78 (GRP78) and other related factors that confer protection from apoptosis. ${ }^{12}$ Intracellularly, this method has been used to study glucose metabolism, but whether GD can be used to clinically access tumor response remains intriguing. ${ }^{13}$ Meanwhile, there has been slow progress in understanding the role of glucose metabolism in the secretion of complex factors that support tumorigenesis and drug resistance.

FOXO3a has been closely implicated in multidrug resistance through the expression of $\mathrm{ABCB} 1$ and PIK3CA in a limited number of malignancies. ${ }^{14-16}$ Highly drug-resistant cancer cells are characterized by anti-apoptotic mechanisms. FOXO3a is phosphorylated by Akt, which inhibits the transactivation of target genes associated with apoptosis and cell proliferation, such as $\mathrm{p} 27^{\mathrm{Kip} 1}$, cyclin $\mathrm{D}$, Bim and Bcl. ${ }^{17,18}$ This inhibition changes the status of FOXO3a as a major target of inactivation by PI3K/Akt. However, little is known regarding how FOXO3a affects the development of resistance in drug-sensitive cells as mediated by therapy/drug-induced cancer cell secretomes. Thus, a better understanding of this process is warranted to improve therapeutic outcomes.

Here we report that FOXO3a deletion and targeted glycolysis block drug-sensitive cancer cell escape from apoptosis and in the development of docetaxel cross-resistance in PacR cells induced by PacR cancer cell-derived secretomes.

\section{MATERIALS AND METHODS}

\section{Parental and drug-resistant cancer cell lines}

Parental A549 human lung cancer and PC-3 human prostate cancer cell lines were obtained from American Type Culture Collection (Manassas, VA, USA) and were cultured in RPMI supplemented with $10 \%$ heat-inactivated fetal bovine serum, $100 \mathrm{U} \mathrm{ml}^{-1}$ penicillin, $100 \mu \mathrm{g} \mathrm{ml} l^{-1}$ streptomycin and $250 \mathrm{ng} \mathrm{ml}^{-1}$ amphotericin B at $37^{\circ} \mathrm{C}$ in a humidified atmosphere with $5 \% \mathrm{CO}_{2}$. PacR and PacR/DCT cell lines were developed from their parental cells (both A549 and PC-3) using stepwise selection for resistance with increasing concentrations of paclitaxel and docetaxel, respectively. The PacR cells were developed over a period of 12 months and were maintained with $100 \mathrm{~nm}$ paclitaxel. The PacR/DCT cells were derived from the PacR cells and were further developed for 4 weeks to have transient crossresistance to docetaxel; the cells were maintained with low concentrations of paclitaxel and docetaxel. PacR-derived cells were characterized as being $>200$-fold more resistant to paclitaxel and to overexpress P-gp/ABCB1. ${ }^{19-21}$

\section{Glucose modification}

For glucose supplementation, adherent cells were maintained in RPMI 1640, which contained $25 \mathrm{~mm}$ glucose supplemented with 5-10\% fetal calf serum. In the experimental culture, the cells were seeded in highglucose medium, and the medium was replaced after $48 \mathrm{~h}$ with either fresh high-glucose medium ( $+25 \mathrm{~mm}$ glucose) or glucose-deprived medium, which contained no glucose ( $0 \mathrm{~mm}$ glucose; Life Technologies, Grand Island, NY, USA). After glucose modification, PacR cells were washed, the medium was replaced with fresh PacR-maintaining cell culture media and cells were further conditioned for $24 \mathrm{~h}$. This protocol ensured that the collected conditioned media (CM) of PacR cancer cells are not glucose-modified media (glucose-enriched or glucose-deprived) but rather the soluble secretomes of PacR cancer cells. The unmodified $\mathrm{CM}$ of PacR cancer cells was considered control secretomes.

\section{RNA silencing and transfection}

For transient gene silencing of FOXO3a, the cells were transfected with stealth scramble (5'-UUCUCCGAACGUGUCACGUTT- $\left.3^{\prime}\right)$ or FOXO3a (5'-AAUGUGACAUGGAGUCCAUUA-3') short interfering RNAs (siRNAs; Santa Cruz Biotechnology, Santa Cruz, CA, USA) using Lipofectamine 3000. The transfectants were subjected to drug treatment and/or assays $48 \mathrm{~h}$ after transfection. ${ }^{22}$

\section{Cytokine profiling}

The interleukin-6 (IL-6), interferon- $\alpha$ (IFN- $\alpha$ ), IFN- $\beta$, IFN- $\lambda 1$, IFN- $\lambda 2$ and tumor necrosis factor- $\alpha$ (TNF- $\alpha$ ) levels were quantified using cytokine-specific enzyme-linked immunosorbent assay kits with pre-coated capture antibodies as per the manufacturer's instructions (Sigma-Aldrich, St Louis, MO, USA). IL-6 levels were preliminarily detected using a Q-Plex Human cytokine screen (16-plex; Quansys Biosciences, Logan, UT, USA). Cell supernatants were prepared, and cytokine release was analyzed as described previously. ${ }^{23}$

\section{Cell growth, cell death, cell cycle and caspase-3/-9 activity assays}

Cell viability was assessed using an MTT (3-(4,5-dimethylthiazol-2-yl)2,5-diphenyltetrazolium bromide) assay as described previously. ${ }^{24}$ Apoptosis was assessed by subjecting the cells to sub- $\mathrm{G}_{1}$ fraction analysis by fluorescence-activated cell sorting (FACS) using a BD FACSCalibur flow cytometer (BD Biosciences, San Jose, CA, USA), a terminal deoxynucleotidyl transferase dUTP nick end labeling (TUNEL) assay (Promega, Madison, MA, USA) and live-cell monitoring coupled with propidium iodide (PI) incorporation into dead/dying cells. ${ }^{20,24,25}$ The analysis of cell cycle distribution was performed by sorting fluorescent cells and evaluating DNA content by FACS. ${ }^{26}$ Caspase- 3 and caspase-9 activity was detected using the CaspACE Assay System (Promega) with alternative Caspase-Glo assays.

\section{Confocal microscopy}

P-gp localization and DNA fluorescence indicated by 4,6-diamidino-2phenylindole were observed using a Zeiss LSM 780 microscope (Carl Zeiss, Jena, Germany). Cells were grown on coverglass-bottomed, $35-\mathrm{mm}$ dishes pre-coated with $0.2-0.5 \%$ gelatin. The cells were then fixed with $4 \%$ paraformaldehyde for $15 \mathrm{~min}$ and blocked with $1 \%$ bovine serum albumin (BSA) for $1 \mathrm{~h}$. The fixed cells were incubated with P-gp primary human antibody at $4{ }^{\circ} \mathrm{C}$ overnight, and then the cells were incubated with fluorescein isothiocyanate-conjugated secondary antibody. 4,6-Diamidino-2-phenylindole was used to counterstain the nuclei.

\section{Determination of ATP content}

Transfected cells were seeded in 96-well plates and were treated with dimethylsulphoxide or the indicated drugs the following day with the 
Table 1 Primer sequences used for qRT-PCR

\begin{tabular}{lll}
\hline Gene & Forward $\left(5^{\prime}\right.$ to $\left.3^{\prime}\right)$ & Reverse $\left(5^{\prime}\right.$ to $\left.3^{\prime}\right)$ \\
\hline FOXO3a & TTCAAGGATAAGGGCGACAG & CAGGTCGTCCATGAGGTTTT \\
AKT & TGGACTACCTGCAACTCGGAGAA & GTGCCGCAAAAGTCTTCATGG \\
TRAIL & AAGGAAGGGCTTCAGTGACC & TGCAGGAGCACTGTGAAGAT \\
VEGF & TTGCCTTGCTGCTCTACCTCCA & GATGGCAGTAGCTGCGCTGATA \\
C-Myc & TACCCTCTCAACGACAGCAG & TCTTGACATTCTCCTCGGTG \\
Cyclin $D$ & AAGCTGTGCATCTACACCGA & CTTGAGCTTGTTCACCAGGA \\
COX-2 & ATCATTCACCAGGCAAATGC & GGCTTCAGCATAAAGCGTTG \\
C-Fos & CGAAGGGACGGAATAAGATG 1 & GCTGCCAAATAAACTCCAG \\
NF- KB & CCCATCATTGCAATAGCAGG & GTTCAAACTTCTGCTCCTGA \\
Survivin & GCAGCACTACTTCTTGACCACC & TCTGCTCCTGAGCATTGACGTC \\
XIAP & TTCAAGAACTGGCCCTTCTTG & TGGCTCCCAGCCTTCCA \\
GAPDH & TGGGACATGGATATACTCAGTTAACAA & GTTAGCCCTCCTCCACAGTGAA \\
\hline
\end{tabular}

Abbreviation: qRT-PCR, quantitative reverse transcription PCR.

indicated stimulus and duration. CellTiter-Glo reagent (Promega) was used to investigate intracellular ATP content according to the manufacturer's instructions. The data acquisition was measured as described previously. ${ }^{27}$

\section{Western blotting}

Proteins were extracted using RIPA (radioimmunoprecipitation assay) lysis buffer containing $20 \mathrm{~mm}$ Tris- $\mathrm{HCl}$ ( $\mathrm{pH} 7.5$ ), 1\% Triton X-100, $150 \mathrm{~mm}$ sodium chloride, $10 \%$ glycerol, $1 \mathrm{~mm}$ sodium orthovanadate, $50 \mathrm{~mm}$ sodium fluoride, $100 \mathrm{~mm}$ phenylmethylsulfonyl fluoride and $1 \times$ protease inhibitor (Roche Applied Science, Manheim, Germany) at $4{ }^{\circ} \mathrm{C}$. The protein concentration of lysates and supernatants was determined using the Bradford method. Western blot analysis was carried out as described previously. ${ }^{20}$ In brief, the cells were lysed by boiling $2 \times$ sample loading buffer ( $250 \mathrm{~mm}$ Tris- $\mathrm{HCl}(\mathrm{pH} 6.8), 4 \%$ SDS, $10 \%$ glycerol, $0.006 \%$ bromophenol blue, $2 \% \beta$-mercaptoethanol, $50 \mathrm{~mm}$ sodium fluoride and $5 \mathrm{~mm}$ sodium orthovanadate) and were incubated for $10 \mathrm{~min}$ at $100^{\circ} \mathrm{C}$. Proteins were subjected to SDS-polyacrylamide gel electrophoresis and were transferred onto polyvinylidene difluoride membranes (Millipore, Bedford, MA, USA). The blots were blocked with $5 \%$ BSA or $5-10 \%$ skim milk in Trisbuffered saline containing $0.1 \%$ Tween-20 (TBST) for $1 \mathrm{~h}$ at room temperature; the membranes were then incubated with primary antibodies in $2.5 \%$ BSA or $5 \%$ skim milk overnight at $4{ }^{\circ} \mathrm{C}$. The membranes were washed with TBST and were incubated for $2 \mathrm{~h}$ at room temperature with the corresponding secondary antibodies diluted in $2.5 \%$ BSA or $5 \%$ skim milk. The membranes were washed with TBST and were exposed to enhanced chemiluminescence solution (Intron, Daejeon, Korea). The blots were detected on X-ray film or with an LAS-4000 imaging system (Fuji Film Corp., Tokyo, Japan).

\section{Quantitative real-time RT-PCR}

Total cellular RNA was isolated, and complementary DNA was synthesized using an OmniScript cDNA synthesis kit (Qiagen, Valencia, CA, USA). The samples for quantitative reverse transcription PCR were prepared using GoTaq and SYBR Green (Promega). Threshold cycle (Ct) values were determined, and samples were run on a Real-Time PCR system (Bio-Rad, Hercules, CA, USA) using a thermal profile that we have previously described. ${ }^{28}$ The primer sequences used for analysis are listed in Table 1. Real-time PCR was performed as previously described. ${ }^{29}$

\section{Statistical analysis}

Statistical significance $(P<0.05)$ was assessed using Student's $t$-test or one-way analysis of variance coupled with Dunnett's $t$-test performed using OriginPro 8.0 (OriginLab Corp, Northampton, MA, USA). For all compounds, half-maximal inhibitory concentration values and non-linear regressions were calculated using TableCurve 2D v5.01 (Systat Software, San Jose, CA).

\section{RESULTS}

A549-PacR cancer cell-derived secretomes lowered drug-stimulated release of IFN- $\alpha$, IFN- $\lambda$ and TNF- $\alpha$ in A549 cells

Cytokines contribute to the regulation of drug-sensitive cell protection afforded by drug-resistant secretomes in vivo. ${ }^{30}$ As IFNs and TNF- $\alpha$ accelerate apoptosis in several malignancies, these factors are actively being considered for the development of new treatment modalities. ${ }^{31}$ In this context, the effects of PacR cell secretomes on the release of these cytokines were determined in A549 cells. Initially, the A549-PacR cells were exposed to three different conditions with or without trypsin (stimulation), two conditions with known cytokine-releaseinducing effects (that is, hypoxic or serum starvation conditions) and a control condition (that is, normal culture conditions; Scheme in Figure 1a), to determine the concentration of IL- 6 in the supernatants. Because IL- 6 augments drug sensitivity in lung cancer cells, ${ }^{30}$ we first determined which conditions could induce relatively lower, but still traceable, levels of IL-6 release by A549-PacR cell secretomes. As a result, the levels of IL- 6 were found to be three- to eightfold higher in both the hypoxic and serum starvation conditions compared with normal culture conditions when cells were stimulated with trypsin (Figure 1b). On the basis of these findings, the subsequent cytokine production was determined under normal culture conditions. The production levels of IFN- $\lambda 1$, IFN- $\lambda 2$, IFN- $\alpha$, IFN- $\beta$ and TNF- $\alpha$ were primarily characterized under unstimulated or trypsin-stimulated conditions and variations 
a

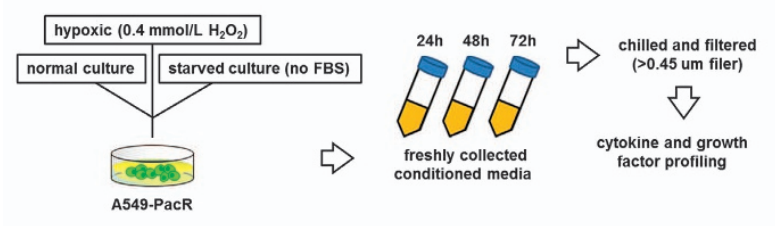

b
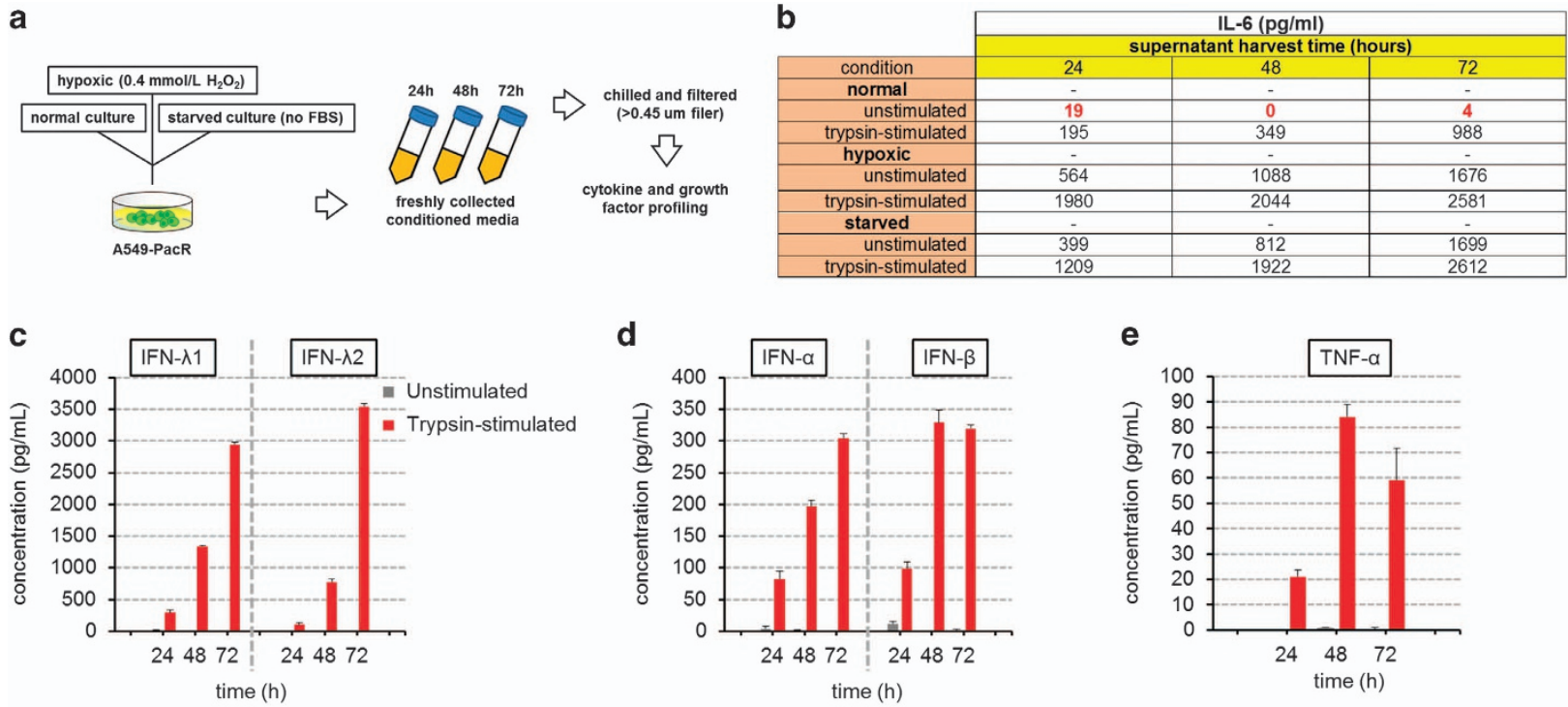

f

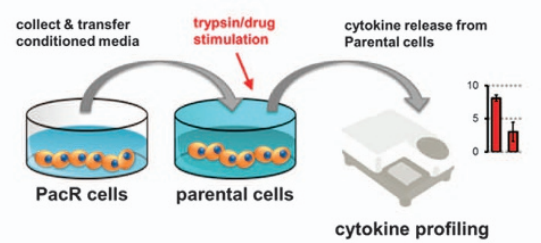

g

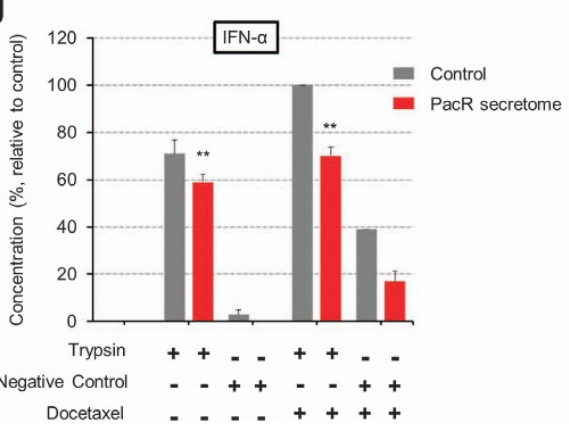

d

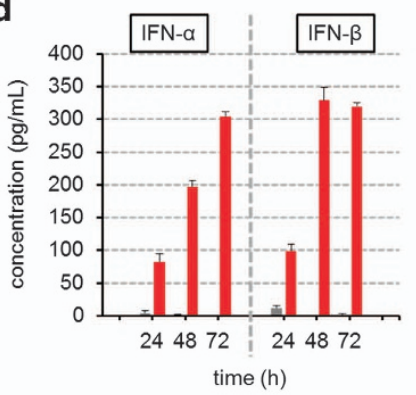

h

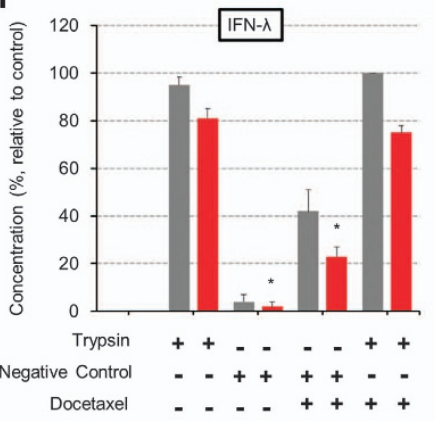

e

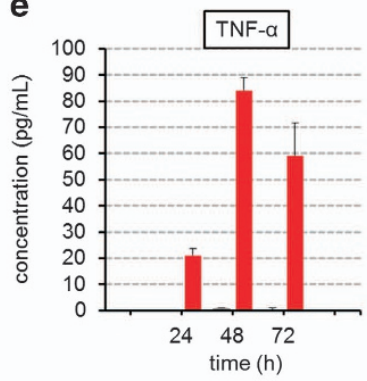

i

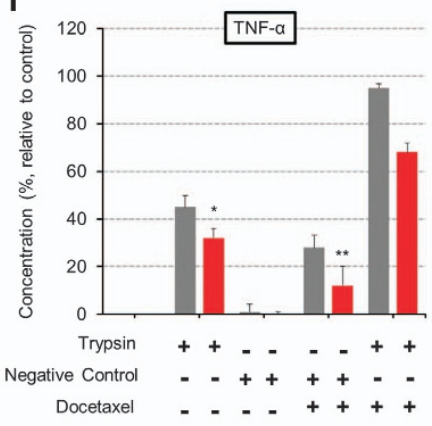

Figure 1 PacR cancer cell-induced secretomes decreased drug-stimulated release of interferon- $\alpha$ (IFN- $\alpha$ ), IFN- $\lambda$ and tumor necrosis factor- $\alpha(T N F-\alpha)$ in parental cells. (a) Schematic work flow of preliminary stimulating conditions and preparation of conditioned media (CM) from A549-PacR cells for cytokine profiling used for assays in b-e. (b) Secretion concentration profile of interleukin-6 from the collected PacR CM after time-dependent harvest in the indicated conditions. Trypsin $(1.2 \mu \mathrm{g} \mathrm{ml-1})$ was used for stimulation. Note that the normal (unstimulated) conditions are highlighted and were used as the control c, d and e. (c-e) Time-dependent release of IFN- $\lambda 1$ (left) and IFN- $\lambda 2$ (right) (c), IFN- $\alpha$ (left) and IFN- $\beta$ (right) (d), and TNF- $\alpha$ (e) following trypsininduced in vitro stimulation with unstimulated conditions as the control. The data are presented as the mean \pm s.d. of the maximal observed cytokine concentration ( $\mathrm{pg} \mathrm{ml}^{-1}$ ). (f) Scheme of A549-PacR CM collection, transfer to and stimulation in adherent A549 cells before cytokine profiling used for assays in $\mathbf{g}-\mathbf{i}$. (g) Influence of docetaxel-stimulation in PacR secretome-induced changes in the IFN- $\alpha(\mathbf{g})$, IFN- $\beta$ (h) and TNF- $\alpha$ (i) release from parental cells. The data are representative of three independent experiments with two biological replicates. The data are presented as the percentage (mean \pm s.d.) ${ }^{*} P<0.05,{ }^{*} P<0.001$ versus the PacR secretome control.

were found in the trypsin-stimulated concentrations at the 24, 48 and $72 \mathrm{~h}$ time points (Figure 1c-e). Further studies were performed to elucidate the regulation of drug (docetaxel)induced cytokine release (Figure 1f). Drug (docetaxel)stimulated induction of the levels of IFN- $\alpha$ (Figure $1 \mathrm{~g}$ ), IFN- $\lambda$ (Figure 1h) and TNF- $\alpha$ (Figure 1i) release was lowered by PacR cell secretomes in A549 cells.
PacR cancer cell-derived secretomes elicited cell outgrowth and escape from TRAIL-induced apoptosis associated with regulation of Akt/FOXO3a signaling in drug-sensitive cancer cells

Our previous study showed that secretomes derived from PacR cells with multiple acquired drug (5-fluorouracil, cisplatin and docetaxel) cross-resistance promoted FOXO3a-directed cell 
growth and drug sensitivity of lung and prostate PacR cancer cells. ${ }^{31}$ To shift the focus onto drug-sensitive cancer cells, we evaluated the effects of PacR cell secretomes on cell growth and apoptosis of drug-sensitive cells. Secreted soluble mediators from drug-resistant cancer cells foster cell growth and regulate the drug-induced cell death response in sensitive cancer cells that feed from this microenvironment, ${ }^{31,32}$ indicating that acquisition of acquired drug resistance from these secreted a

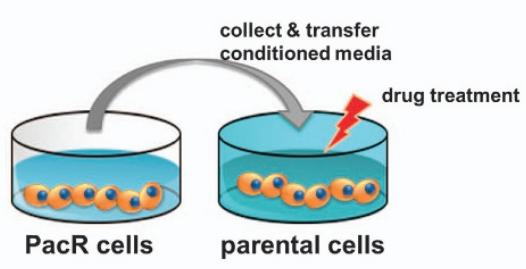

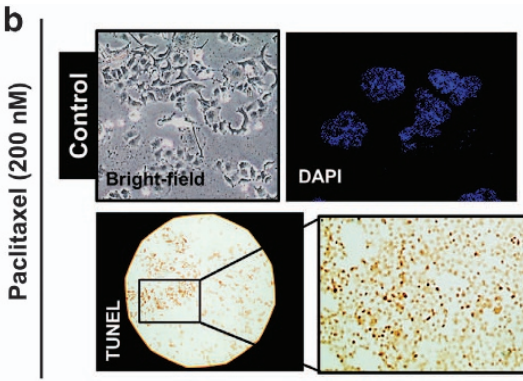

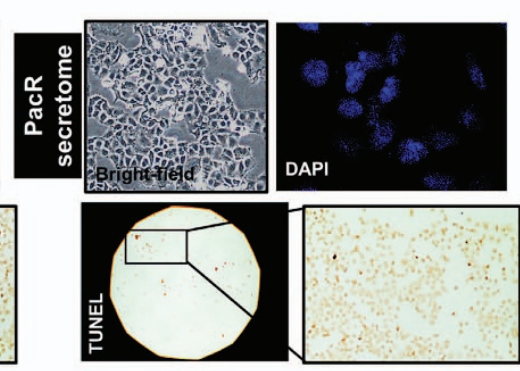

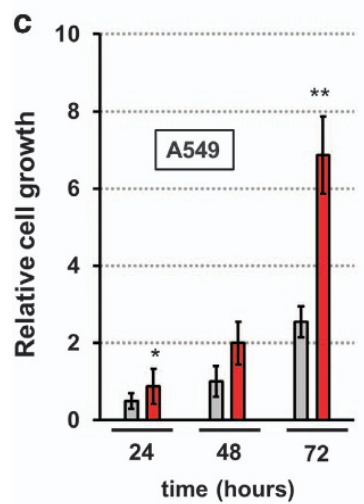

f

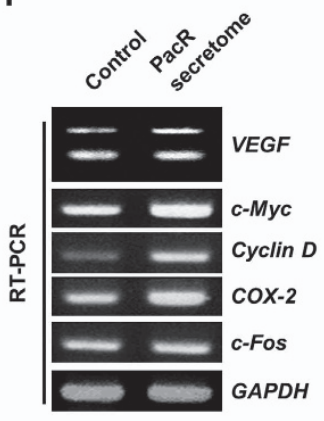

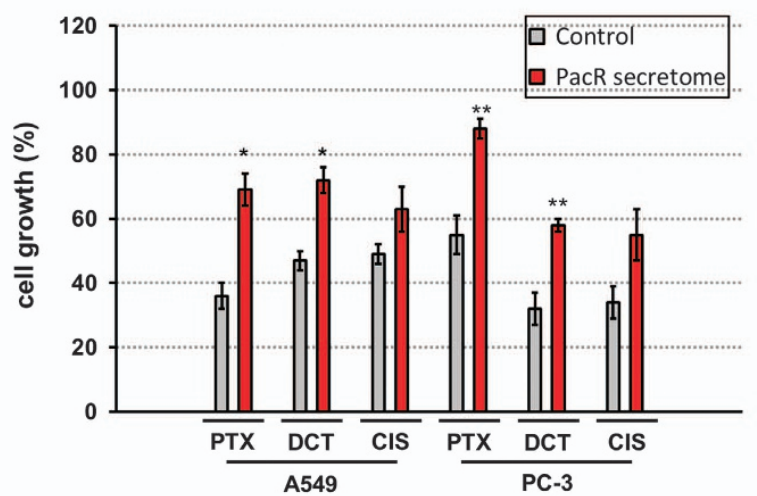

d
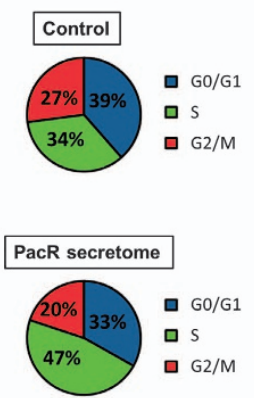

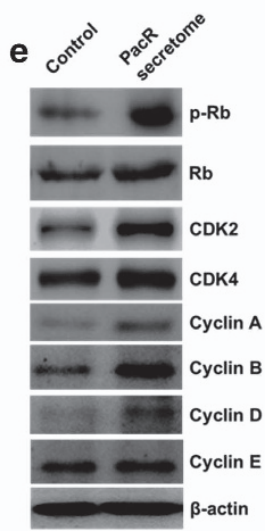

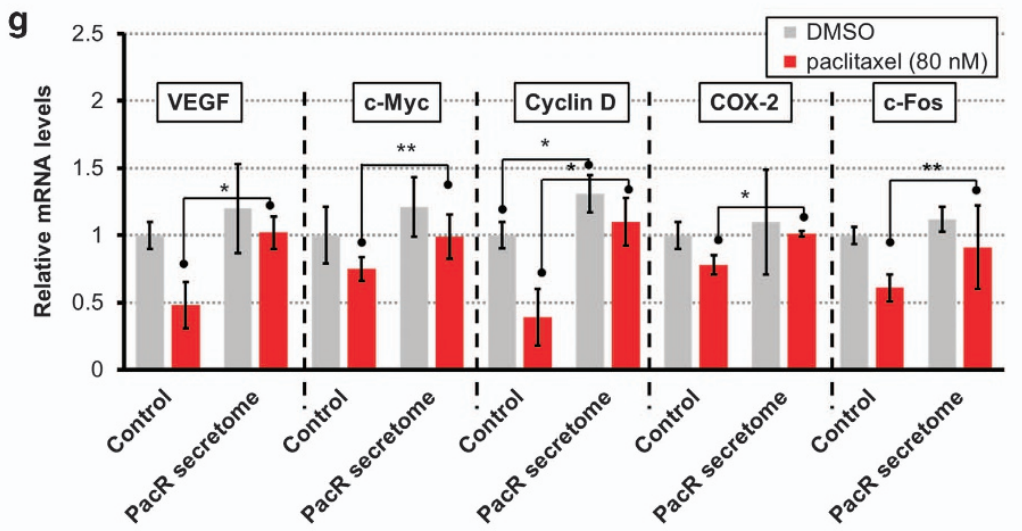

h

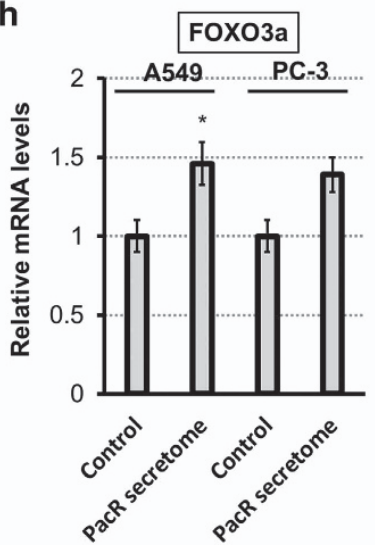

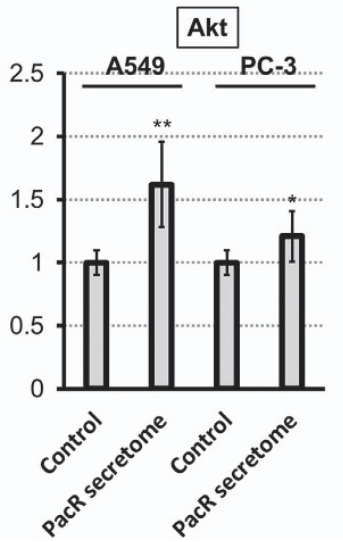

i

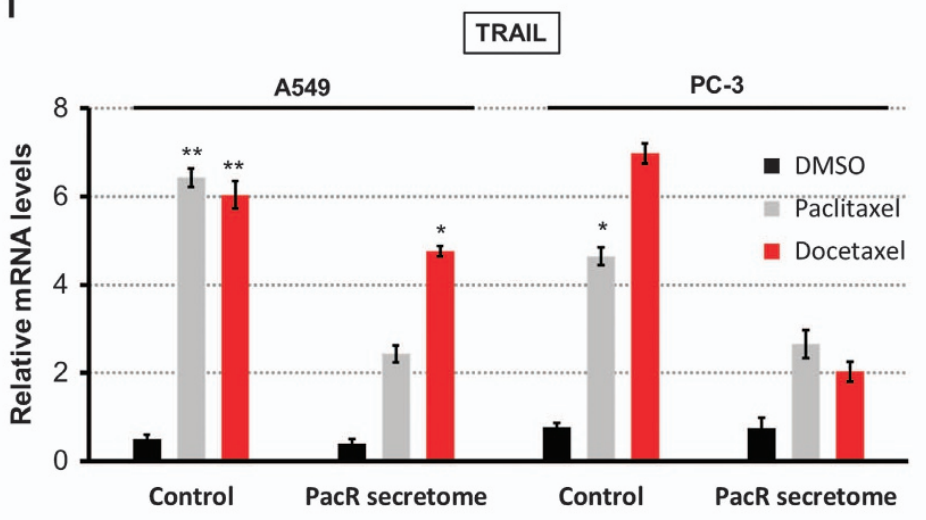


factors, particularly from PacR cancer cells, can be associated with an aggressive phenotype in vitro. ${ }^{33,34}$ Interestingly, we found sustained proliferation of parental A549 cells that were exposed to A549-PacR cell secretomes, even after paclitaxel challenge at $24-48 \mathrm{~h}$ (Supplementary Figure S1). We then determined whether PacR cell secretomes afforded protection from drug-induced cytotoxicity (Scheme in Figure 2a). When A549 and PC-3 cells were exposed to PacR cell secretomes, paclitaxel-induced apoptotic cell death (for example, deformed epithelial appearance, 4,6-diamidino-2-phenylindole-stained nuclei and TUNEL-positive nuclei) was inhibited in A549 cells (Figure $2 \mathrm{~b}$ ), cell growth was promoted in a time-dependent manner (left panel of Figure 2c), and chemotherapeuticsinduced cell growth inhibition was alleviated (right panel of Figure 2c). In addition, the exposure of A549 cells to PacR cell secretomes produced an increase of $13 \%$ in $S$ phase and a minimal decrease in $G_{0} / G_{1}$ phase in these cells, which were accompanied by profound regulation of cell cycle-related proteins (Figure 2d and e). Moreover, PacR cell secretomes also modulated paclitaxel-induced gene regulatory responses observed in the expression levels of VEGF, c-Myc, cyclin D, COX-2 and c-Fox in A549 cells (Figure $2 \mathrm{f}$ and g). These genes have long been described as regulators of drug resistance, ${ }^{35,36}$ thus these findings might be positively correlated with the cell growth-promoting effects of PacR cell secretomes.

The activation of FOXO3a through Akt regulates TRAILtargeting signals to affect cell death in drug-resistant cancer cells. ${ }^{37,38}$ Accordingly, targeting strategies that use molecules to modulate FOXO3a are under active investigation..$^{39,40}$ In the present study, we found that PacR-derived cell secretomes upregulated the gene expression of FOXO3a and Akt in both A549 and PC-3 cells (Figure 2h). In addition, to further determine whether Akt controls FOXO3a expression in these cells, we initially identified the ubiquitination status of FOXO3a in the presence of the Akt inhibitor LY294002. LY294002 induced FOXO3a ubiquitination with the concurrent inhibition of FOXO3a protein expression. Furthermore, LY294002 induced apoptosis of A549 cells exposed to PacR cell secretomes (Supplementary Figure S2). We then examined TRAIL expression in these cells after treatment with apoptosis-inducing concentrations of paclitaxel and docetaxel. Exposure to PacR cell secretomes led to decreased drug-induced TRAIL gene expression in both A549 and PC-3 cells (Figure 2i). These results suggest that protection from drug-induced apoptosis mediated by TRAIL correlates with the acquired resistance-promoting effects of the PacR secretomes on drug-sensitive parental cells, which may involve Akt/FOXO3a. These findings are consistent with the previously reported increased expression and nuclear accumulation of FOXO3a despite enhanced PI3K/Akt activity in drug-resistant K562 cells. ${ }^{15}$

\section{Glucose-deprived PacR cell-derived secretomes altered their cell growth-promoting activity via FOXO3a-mediated ATP depletion in drug-sensitive cancer cells}

The acquisition of drug-resistant cancer cell phenotypes has been associated with increased dependence on glucose for survival and stress defense. ${ }^{41,42}$ Therefore, we investigated the underlying mechanisms of the protective effects of PacR cell secretomes against drug-induced apoptosis in A549 and PC-3 cells. To this end, glucose metabolism (withdrawal or supplementation) was modified in PacR cells before the CM harvest and exposure to parental cancer cells (Scheme in Figure 3a). We also employed FOXO3a RNA interference to observe the regulatory mechanism of FOXO3a in Akt-induced glycolysis. ${ }^{43}$ The selected stealth siRNA for FOXO3a effectively suppressed the expression of FOXO3a messenger RNA and protein levels (Figure 3b). Glucose-deprived PacR cell-derived secretomes substantially altered the effect of secretomes on growth promotion, as observed by the time-dependent inhibition of cell growth, which was similar when FOXO3a transcription was suppressed both in A549 and PC-3 cells (Figure 3c). The susceptibility of tumor cells to cell death is known to be regulated by glucose metabolism and ATP coordination. Glucose withdrawal can lead to ATP depletion, possibly leading to apoptosis. ${ }^{44}$ On the basis of this hypothesis, we first determined whether glucose-deprived PacR cell secretomes modified the ATP production in parental cells. Glucosedeprived PacR cell secretomes depleted ATP production in a time-dependent manner in both A549 and PC-3 cells when the

Figure 2 PacR cancer cell-induced secretomes promote cell proliferation and escape from TRAIL-induced apoptosis in parental cancer cells and the involvement of FOXO3a/Akt signaling. (a) Scheme of PacR conditioned media collection, transfer and drug treatment in A549 cells used for assays in b-g. (b) Cell phase-contrast, fluorescence images of cell morphologies and TUNEL-positive nuclei of adherent A549 cells after exposure to control or PacR secretomes with subsequent paclitaxel treatment (200 nm) for $24 \mathrm{~h}$. (c) Relative cell growth rates of $\mathrm{A} 549$ cells after time-dependent exposure to control or PacR secretomes (left panel) with subsequent paclitaxel (PTX, $60 \mathrm{~nm}$ ), docetaxel (DCT, $60 \mathrm{~nm}$ ) or cisplatin (CIS, $60 \mu \mathrm{m}$ ) treatment for $24 \mathrm{~h}$. The data are presented as the percentage (mean $\pm \mathrm{s} . \mathrm{d}$. ). (d) Analysis of cell cycle distribution in A549 cells after exposure to control or PacR secretomes. Cell cycle distribution was analyzed by flow cytometry. (e) Effects of PacR secretomes on the expression of cell cycle regulatory proteins. $\beta$-Actin was used as the loading control. Fresh medium replacement was used as a control. (f, $\mathbf{g}$ ) PCR transcripts (left) and messenger RNA (mRNA) gene expression levels (right) of growth-related genes VEGF, c-Myc, cyclin D, COX-2 and c-Fos in A549 cells after exposure to control or the PacR secretome. (h) mRNA gene expression of FOXO3a (left panel) and Akt (right panel) in A549 and PC-3 cells after exposure to the control or PacR secretome. (i) TRAIL mRNA gene expression in A549 and PC-3 cells after exposure to the control or PacR secretomes with subsequent PTX (200 nm) and DCT $(150 \mathrm{~nm})$ treatments for $24 \mathrm{~h}$. GAPDH mRNA levels were used for normalization. The data are presented as the mean fold changes \pm s.d. relative to the control. The results are representative of two $(\mathbf{h})$ and three $(\mathbf{a}-\mathbf{d}, \mathbf{f}, \mathbf{g})$ independent experiments. ${ }^{*} P<0.05$, $* * P<0.001$ versus the PacR secretome control. 
a

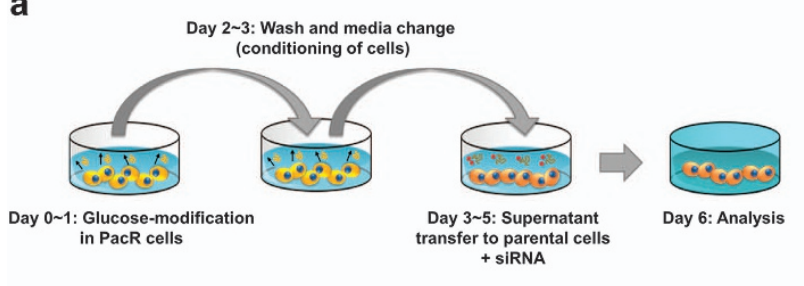

b
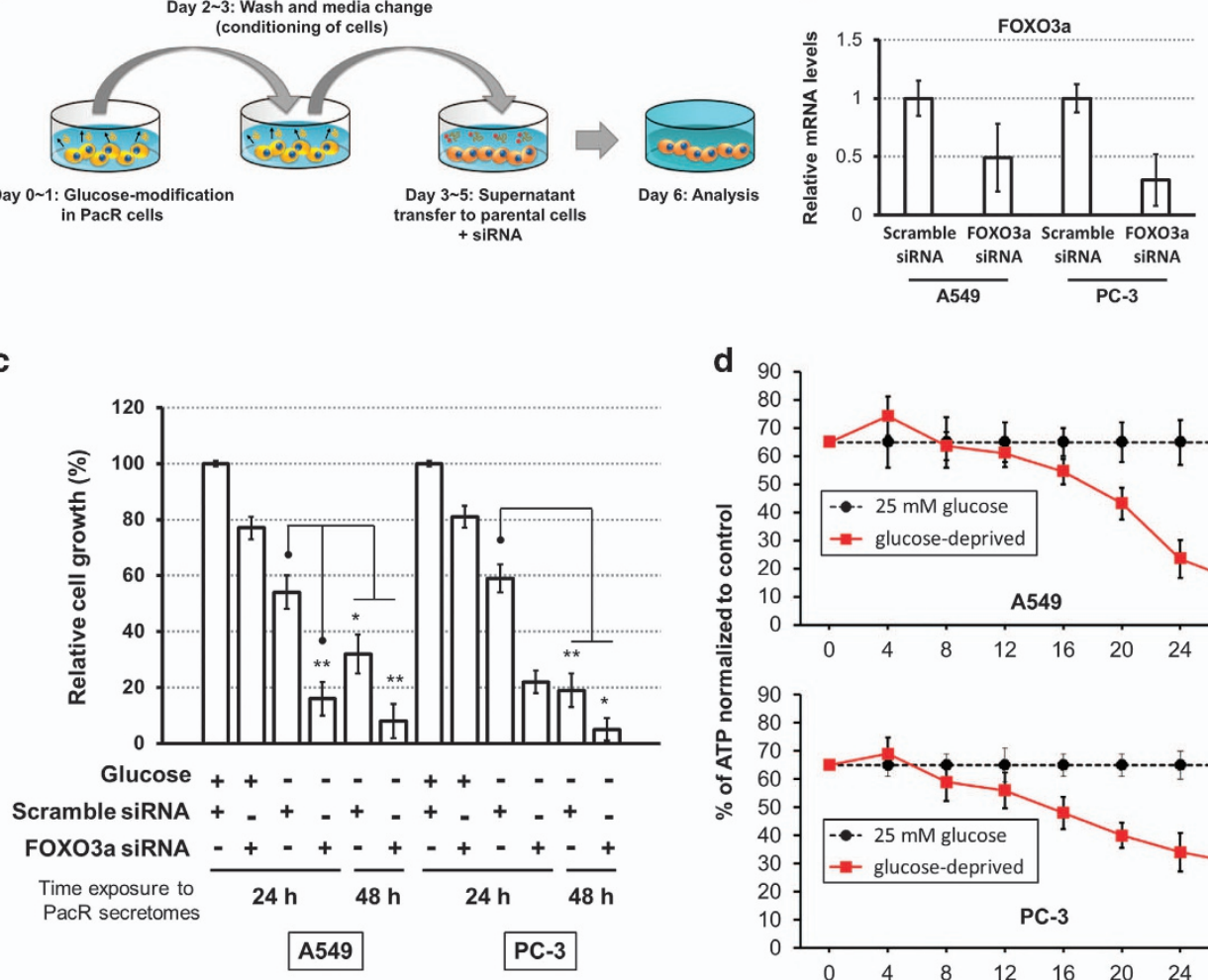

d

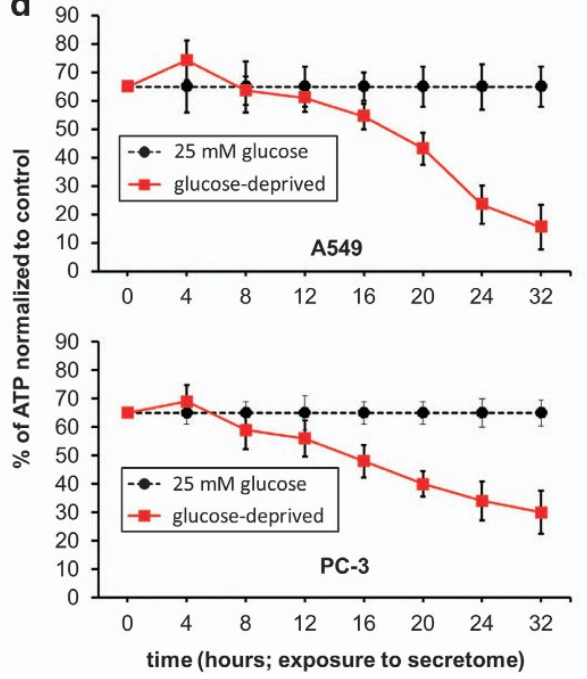

e

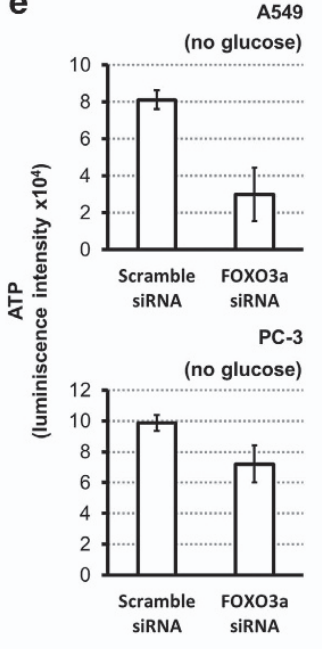

Figure 3 Glucose modification-induced changes in PacR cancer cell-induced secretome-mediated regulation of cell growth and ATP production involve FOXO3a. (a) Scheme of glucose deprivation in PacR and FOXO3a short interfering RNA (siRNA) in adherent parental cells used in assays (c-e). (b) siRNA experiments with A549 and PC-3 cells. Cells were transfected with negative control siRNA or FOXO3a siRNA for $48 \mathrm{~h}$. The levels of FOXO3a messenger RNA (mRNA) are indicated by real-time PCR (left panel). GAPDH mRNA levels were used for normalization. The cell lysates were immunoblotted with FOXO3a antibody (right panel). $\beta$-Actin was used as the loading control. The data are represented as the mean \pm s.e.m. (c) Cell viability of A549 and PC-3 cells after exposure to the glucose-modified PacR secretomes for 24 or $48 \mathrm{~h}$ following RNA interference (RNAi) experiments. The data are presented as the percentage (mean \pm s.d.). (d) Time-dependent ATP production rate of A549 and PC-3 cells after scheme in A. Glucose (25 mm)-supplemented PacR secretomes were set as the dependent factor for the levels of glucose-starved PacR secretomes. ATP levels were normalized to control. The data are presented as percentage (mean \pm s.d.). (e) Intracellular ATP luminescence intensities of A549 and PC-3 cells after exposure to glucosedepleted PacR secretomes for $48 \mathrm{~h}$ following subsequent indicated RNAi experiments. The data are presented as the mean $\pm \mathrm{s}$.d. The results are representative of three independent experiments. ${ }^{*} P<0.05,{ }^{*} P<0.001$ versus PacR secretome controls.

levels were normalized with those of the glucose-supplemented cells (Figure 3d), whereas ATP levels were totally inhibited when normalized to those of control (unmodified) cells (data not shown). We also found that the transient silencing of FOXO3a caused further depletion of ATP in both A549 and PC-3 cells after exposure to glucose-deprived PacR cell secretomes (Figure 3e), suggesting that ATP depletion is highly correlated with cell growth inhibition or, possibly, apoptosis. The results of experiments with drug-sensitive parental cells that were exposed to unmodified control PacR cancer cell-derived secretomes are presented in Supplementary Figure S3.

Glucose modification in PacR cancer cells promoted the secretion of soluble factors that direct cell death via FOXO3a in drug-sensitive cancer cells

By modifying glucose utilization in cancer cells, several secreted factors have been reported to promote the metastatic niche and protection from cell death-inducing molecules. ${ }^{45,46}$ To identify whether glucose modification in PacR cells can regulate the secretion of factors that mediate cell death in drug-sensitive cancer cells and determine the involvement of FOXO3a, FOXO3a RNA interference was employed to detect the effect on cell viability. Exposure to glucose-deprived PacR cell secretomes led to an increase in the apoptotic sub- $\mathrm{G}_{1}$ phase fraction with the deletion of FOXO3a, indicating the likelihood of paclitaxel-induced apoptosis (Figure $4 \mathrm{a}$ ). In addition, exposure to glucose-deprived PacR cell secretomes also led to increased caspase- 3 and caspase- 9 activity with FOXO3a deletion, exacerbating the incidence of apoptosis in drug-sensitive cancer cells (Figure $4 \mathrm{~b}$ ). This finding suggests the probable involvement of additional cell death signals regulating this effect. Glucose-deprived PacR cell secretomes led to minimally induced PARP cleavage in A549 cells, and FOXO3a suppression did not seem to affect this outcome, even 
a

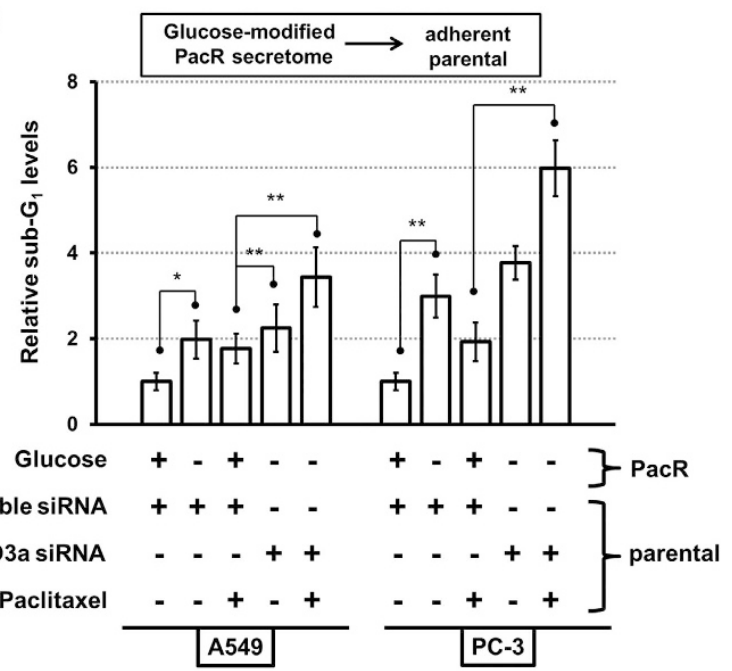

b

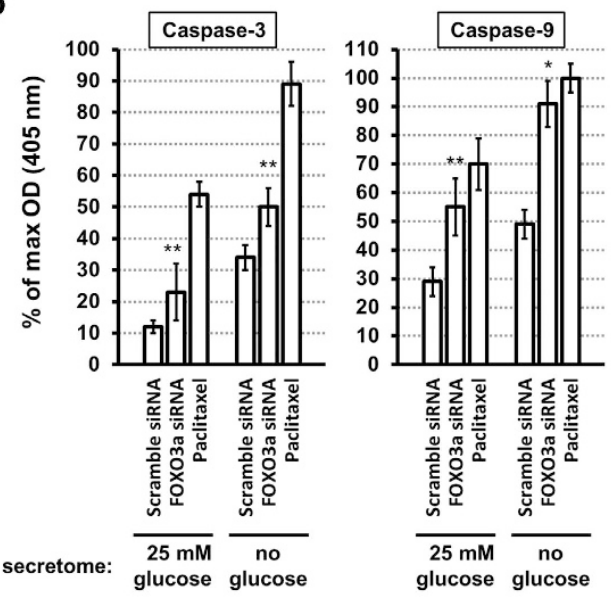

C
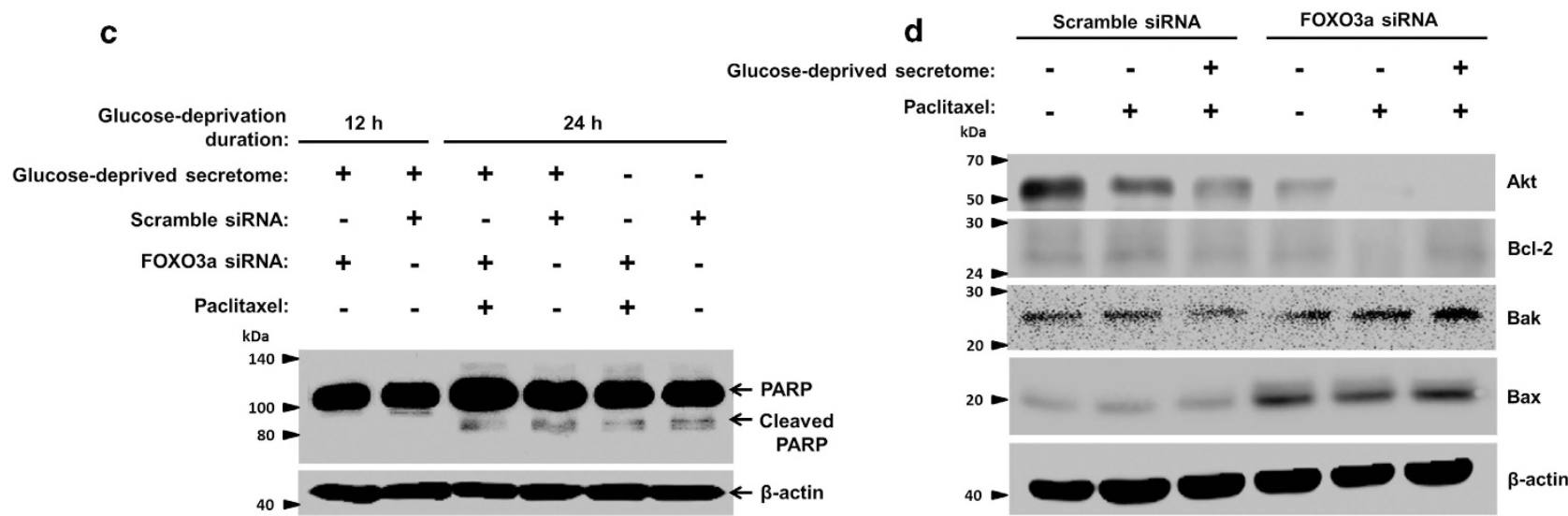

Figure 4 Targeted glycolysis in PacR cancer cells promotes the secretion of factors regulating apoptosis in adherent parental cancer cells. (a) Sub-G $\mathrm{G}_{1}$ population of A549 and PC-3 cells after exposure to glucose-modified (+, 25 mM glucose; -, glucose deprived) PacR cell secretomes following subsequent RNA interference (RNAi) experiments and paclitaxel ( $300 \mathrm{~nm}$ ) treatment for $24 \mathrm{~h}$. The sub-G fraction was quantified by FACS analysis. The data are presented as relative values (mean \pm s.d.). (b) Caspase-3 (left panel) and caspase-9 (right panel) activities of A549 cells after exposure to glucose-modified PacR secretomes following subsequent RNAi experiments and paclitaxel (300 nm) treatment for $24 \mathrm{~h}$. Caspase activities were quantified by measuring the maximum optical density (max OD) values of proluminogenic caspase-3/9 substrates after transfection experiments. The data are presented as the percentage (mean \pm s.d.). (c) Accumulation of cleaved PARP and PARP expression in A549 cells after time-dependent exposure to the glucose-deprived (+, withdrawn; -, control) PacR cell secretomes following subsequent RNAi experiments and paclitaxel (200 nm) treatment for $24 \mathrm{~h}$. $\beta$-Actin was used as the loading control. Fresh medium replacement was used as the control. (d) Protein expression of Akt, Bcl-2, Bak and Bax in A549 cells after exposure to glucose-deprived (+, withdrawn; -, control) control or PacR secretomes following subsequent RNAi experiments and paclitaxel (200 nm) treatment for $24 \mathrm{~h}$. $\beta$-Actin was used as the loading control. Fresh medium replacement was used as the control. The results are representative of three independent experiments. siRNA, short interfering RNA.

with paclitaxel stimulation (Figure 4c). Moreover, FOXO3a regulated the induced downregulation of Akt, and activation of Bak and Bax in A549 cells upon exposure to glucose-deprived PacR cell secretomes (Figure 4d). This result provides evidence that the apoptosis-promoting effect of glucose-deprived PacR cell secretomes in drug-sensitive cells may be mediated, at least in part, by FOXO3a.

\section{Regulation of apoptosis in PacR cells with docetaxel} cross-resistance was mediated by glucose-modified cell secretion factors via $\mathrm{FOXO}$ a

To examine the mediators of apoptosis in relation to the acquisition of cross-resistance to docetaxel in PacR cells, we employed our established PacR/DCT cell lines derived from PacR variants. PacR/DCT cells have transient cross-resistance to docetaxel yet retain substantial resistance to paclitaxel. We assessed the effects of glucose modifications in PacR cell secretomes on adherent PacR/DCT cells based on high P-gp/ABCB1 dependence of acquired cross-resistance. We also examined the role of FOXO3a in apoptosis in these resistant variants. Active utilization of glucose from the microenvironment by drug-resistant tumors has been previously reported. ${ }^{47}$ Furthermore, side populations of tumor cells with acquired resistance are also described to have elevated glycolytic activity, ${ }^{48}$ indicating higher glucose uptake and lower oxygen consumption, ${ }^{31,47}$ which increases the intensity of 
a
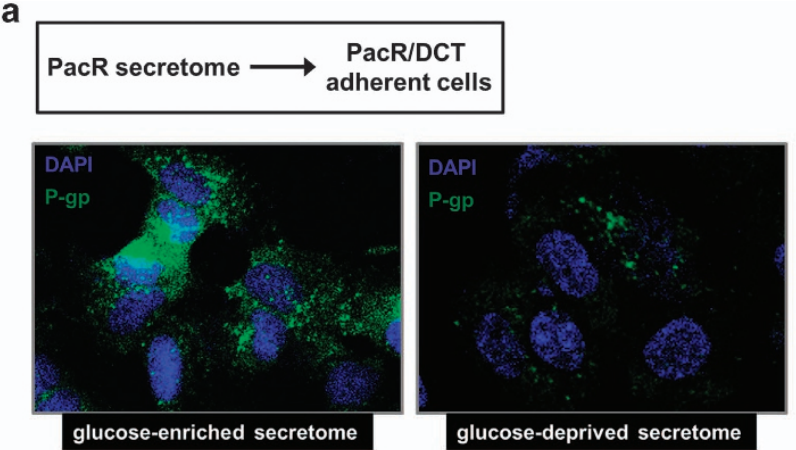

b

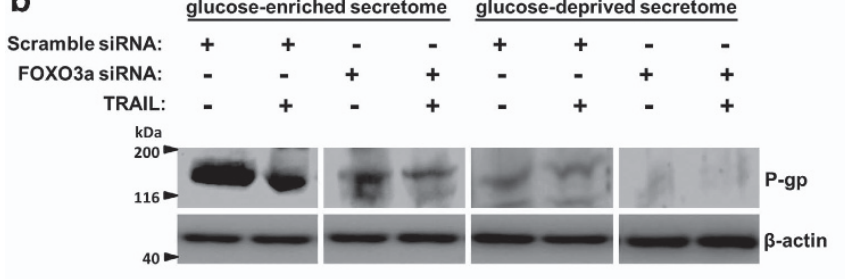

C
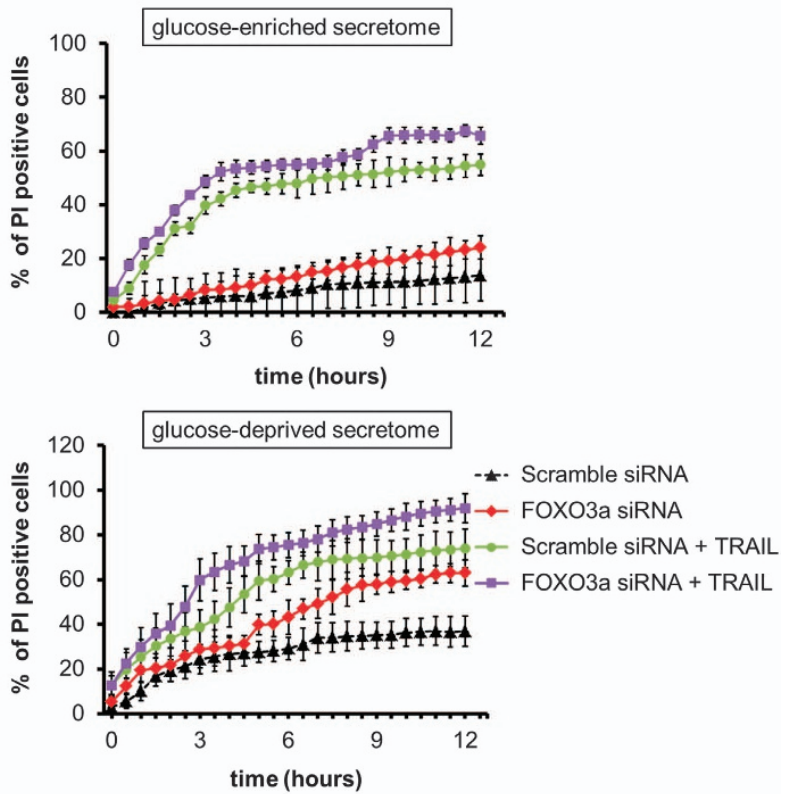

d

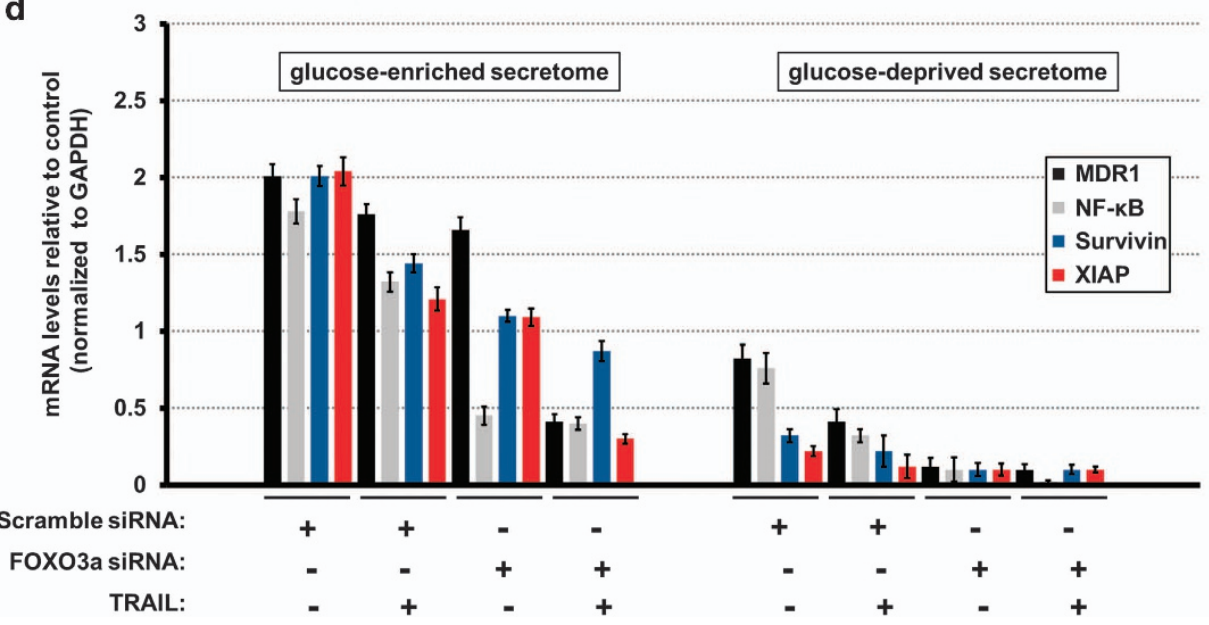

Figure 5 Targeted glycolysis regulates P-glycoprotein (P-gp)/ABCB1-associated acquired drug resistance and apoptosis in cancer cells grown under exposure to PacR cancer cell-induced secretomes. (a) P-gp (green) distribution in A549-PacR/DCT cells after exposure to glucose-modified PacR cell secretomes. P-gp localization was observed by confocal microscopy. The nuclei were stained with 4,6-diamidino-2-phenylindole (DAPI; blue). Images are representative of three independent experiments. Images were scaled at $40 \mu \mathrm{m}$. (b) P-gp protein expression of A549-PacR/DCT cells after exposure to glucose-modified PacR cell secretomes following subsequent RNA interference (RNAi) experiments and TRAIL (400 $\mathrm{ng} \mathrm{ml}^{-1}$ ) treatment for $24 \mathrm{~h}$. $\beta$-Actin was used as the loading control. (c) Apoptotic live-cell monitoring coupled with PI incorporation into dead/dying cells after A549-PacR/DCT cells were exposed to glucose-modified PacR cell secretomes following subsequent RNAi experiments and TRAIL (300 $\mathrm{ng} \mathrm{ml}^{-1}$ ) treatment for $24 \mathrm{~h}$. The percent of PI incorporation was monitored time-dependently after transfection and treatment procedures. Non-overlapping PI-stained cells were counted according to the indicated time intervals, and the total number of PI-positive nuclei was scored. All data were normalized to the first time point $(t=0)$ for convenient comparisons. The data are presented as the percentage (mean \pm s.d.). (d) Messenger RNA (mRNA) gene expression of ABCB1 (MDR1), NF-KB, survivin and XIAP in A549-PacR/DCT cells after exposure to glucose-modified PacR secretomes following subsequent RNAi experiments and TRAIL (400 $\mathrm{ng} \mathrm{m}^{-1}$ ) treatment for $24 \mathrm{~h}$. GAPDH mRNA levels were used for normalization. The data are represented as the mean \pm s.e.m. siRNA, short interfering RNA.

drug resistance. This information prompted us to compare glucose-enriched (25 mm glucose+) and glucose-deprived PacR cell secretomes and their effects on P-gp/ABCB1-associated docetaxel cross-resistance and apoptosis (Scheme on upper panel Figure 5a). P-gp distribution in the membrane and cytoplasm of A549-PacR/DCT cells was subdued in response to exposure to glucose-deprived PacR cell secretomes, whereas cells exposed to glucose-enriched PacR cell secretomes retained their P-gp distribution (Figure 5a). Furthermore, the inhibition of P-gp was apparent in A549-PacR/DCT cells after they were 

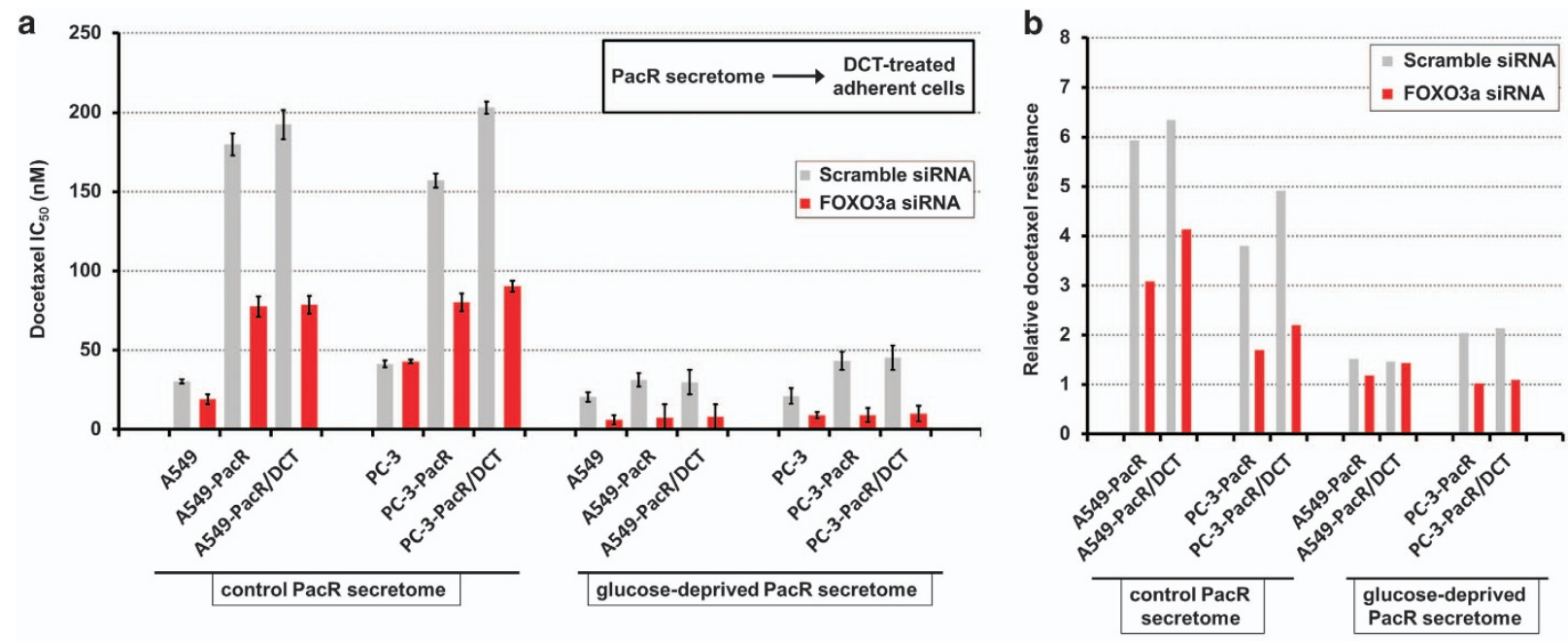

C
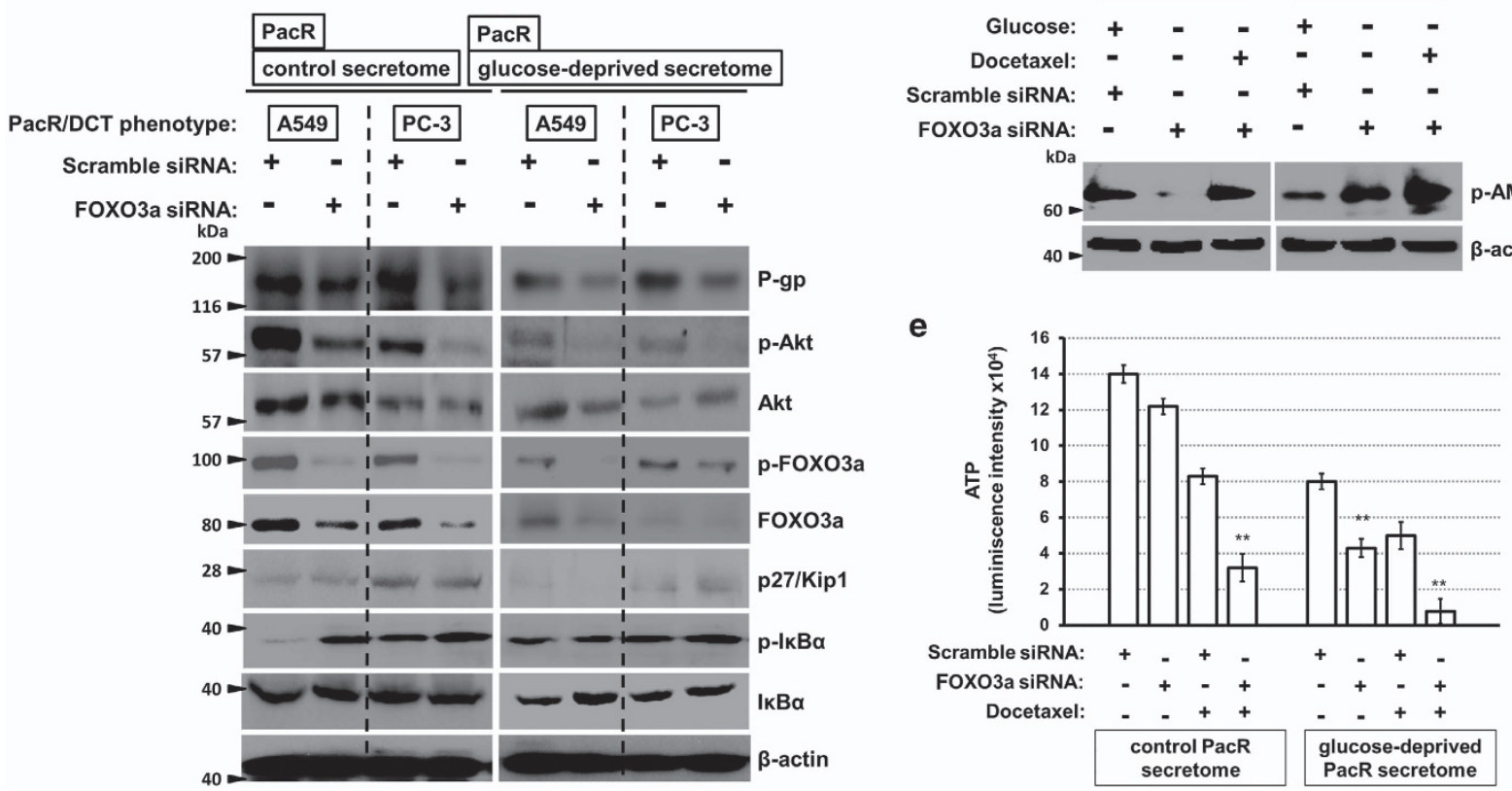

Figure 6 Targeted glycolysis regulates PacR cancer cell-induced secretome-mediated promotion of docetaxel cross-resistance in PacR/DCT cells through FOXO3a. (a) Sensitivity screen of docetaxel in parental, PacR and PacR/DCT phenotypes of A549 and PC-3 cells after exposure to the non (control)- or glucose-deprived PacR cell secretome. Docetaxel half-maximal inhibitory concentration (IC 50 ) was identified by testing five different concentrations of the drug in a $48 \mathrm{~h}$ treatment window. Cell viability was assessed by MTT assay. The data are presented as percentages (mean \pm s.d.). (b) Relative resistance/cross-resistance of docetaxel in PacR and PacR/DCT phenotypes of A549 and PC-3 cells were calculated by dividing docetaxel I $\mathrm{C}_{50}$ values in PacR and PacR/DCT variants by parental cell values. (c) Protein expression of P-glycoprotein (P-gp), p-Akt, Akt, p-FOXO3a, FOXO3a, p27/Kip1, p-IкB $\alpha$ and $1 \kappa B \alpha$ in the PacR/DCT phenotype of A549 and PC-3 cells after exposure to the control or glucose-deprived PacR cell secretomes following subsequent RNA interference (RNAi) experiments. (d) Phospho-AMPK- $\alpha$ expression in A549-PacR/DCT and PC-3-PacR/DCT cells after exposure to PacR cell secretomes for $24 \mathrm{~h}$ following subsequent glucose modification (+, $25 \mathrm{~mm}$ glucose in medium; -, glucose-depleted medium), RNAi experiments and docetaxel (40 nm) treatment in cultures of adherent cells. (e) Intracellular ATP luminescence intensities of A549-PacR/DCT cells after exposure to the control or glucose-deprived PacR cell secretomes following subsequent RNAi experiments and docetaxel (60 nm) treatment for $24 \mathrm{~h}$. The data are presented as the percentage (mean \pm s.d.). The results are representative of two (a) and three (b-e) independent experiments. ${ }^{*} P<0.05,{ }^{*} P<0.001$ versus control. siRNA, short interfering RNA.

exposed to glucose-deprived PacR cell secretomes with or without TRAIL treatment, whereas glucose enrichment led to a maintained expression except after RNA interference transfection. Transfection with FOXO3a siRNA intensified this suppression compared with that of control cells (Figure 5b). To correlate this finding with apoptosis, we exposed both A549-PacR/DCT and PC-3-PacR/DCT cells to glucoseenriched or glucose-deprived PacR cell secretomes and 


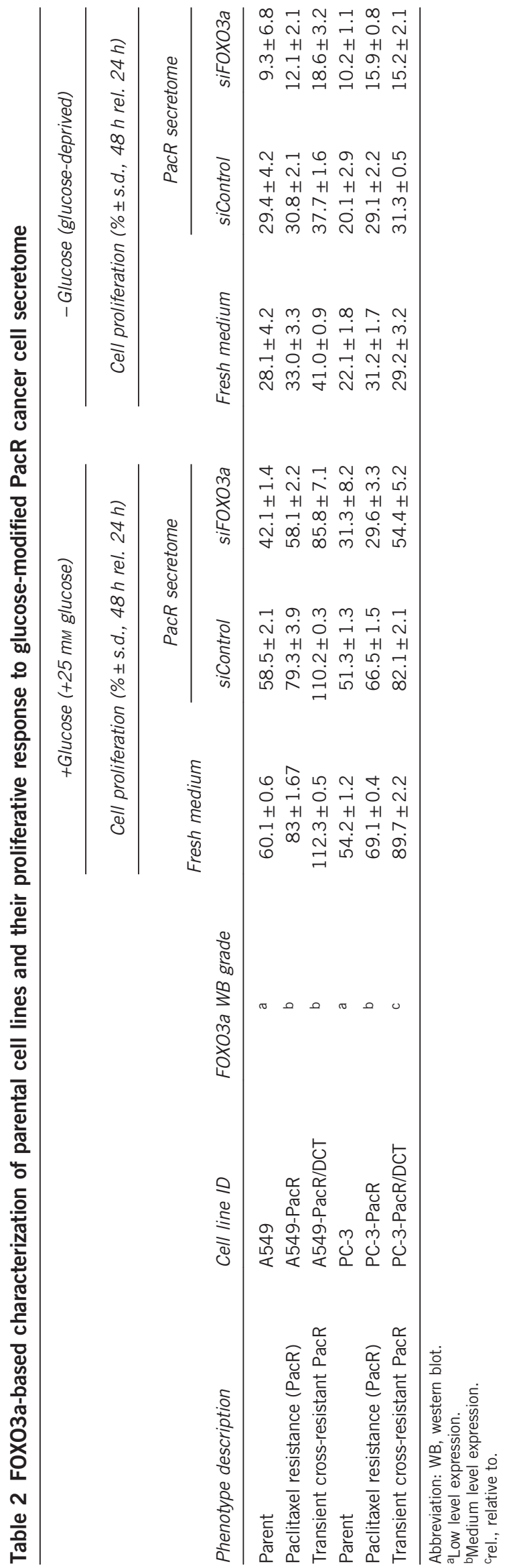

transfected the cells with FOX3a siRNA. PI can fluorescently label the nuclei of necrotic or late-stage apoptotic cells because PI is normally membrane impermeable. ${ }^{49}$ Therefore, it has been shown that the increase in the number of PI-stained nuclei over time can be used as a metric for cell death..$^{50}$ Images of live cells were acquired every $0.5 \mathrm{~h}$ over a total of $12 \mathrm{~h}$, and the total number of PI-positive nuclei was determined and normalized to 1 (time; $t=0$ ). In A549-PacR/DCT and PC-3-PacR/DCT cells exposed to glucose-enriched PacR cell secretomes, minimal differences were observed between the control and the FOXO3a siRNA-transfected cells over time; this finding was also consistent with the TRAIL-stimulated group (upper panels of Figure $5 \mathrm{c}$ and Supplementary Figure S4). However, glucose-deprived PacR cell secretomes induced higher levels of apoptotic cells, and significant differences can be observed between the control and FOXO3a siRNA-transfected cells: TRAIL-stimulated, FOXO3a siRNAtransfected cells showed relatively higher levels of apoptosis than did TRAIL-stimulated control cells (lower panels of Figure 5c; Supplementary Figure S4). To confirm these observed differences in cell death, we measured the gene expression levels of cell death-related signals and $\mathrm{ABCB} 1$ (MDR1) under the same conditions in A549-PacR/DCT cells. Exposure to glucose-deprived PacR cell secretomes inhibited the gene expression levels of $\mathrm{ABCB} 1, \mathrm{NF}-\mathrm{\kappa B}$, survivin and XIAP, and further regulated their TRAIL-mediated levels in A549-PacR/DCT cells compared with those observed after exposure to glucose-enriched PacR cell secretomes, the deletion of FOXO3a intensified this inhibitory effect (Figure 5d). These results indicated that the knockdown of FOXO3a effectively mediated the induction of cell death, even after exposure to both types of glucose-modified PacR cell secretomes.

Targeted glycolysis involved FOXO3a/Akt in the regulation of docetaxel cross-resistance in PacR cancer cells induced by their secretomes

Modifications in metabolic energy produced by glycolysis are essential contributors to the progression of multidrug resistance in cancer cells. ${ }^{51}$ The deregulation of signals responsive to GD in drug-resistant cancer cells has been reported previously and has been shown to counteract drug-induced toxicity. ${ }^{52}$ In addition, transcriptome analysis of these signals in drug-sensitive cells in response to treatmentinduced tumor secretomes has also been described. ${ }^{53}$ Therefore, we next determined whether the cell secretomes derived from A549-PacR and PC-3-PacR variants mediate the sensitivity to docetaxel in both PacR and PacR/DCT cells. Interestingly, we found that glucose modification in these cell secretomes affected the sensitivity to docetaxel in both PacR and PacR/DCT cells. In addition, FOXO3a siRNA enhanced the sensitivity to docetaxel in the same experimental conditions (Figure 6a). Relative acquired resistance to docetaxel was calculated based on the half-maximal inhibitory concentration levels, which further confirmed the docetaxel sensitivity results obtained from cells exposed to the secretomes of PacR cells (Figure 6b). Similarly, in our FOXO3a-based characterization 


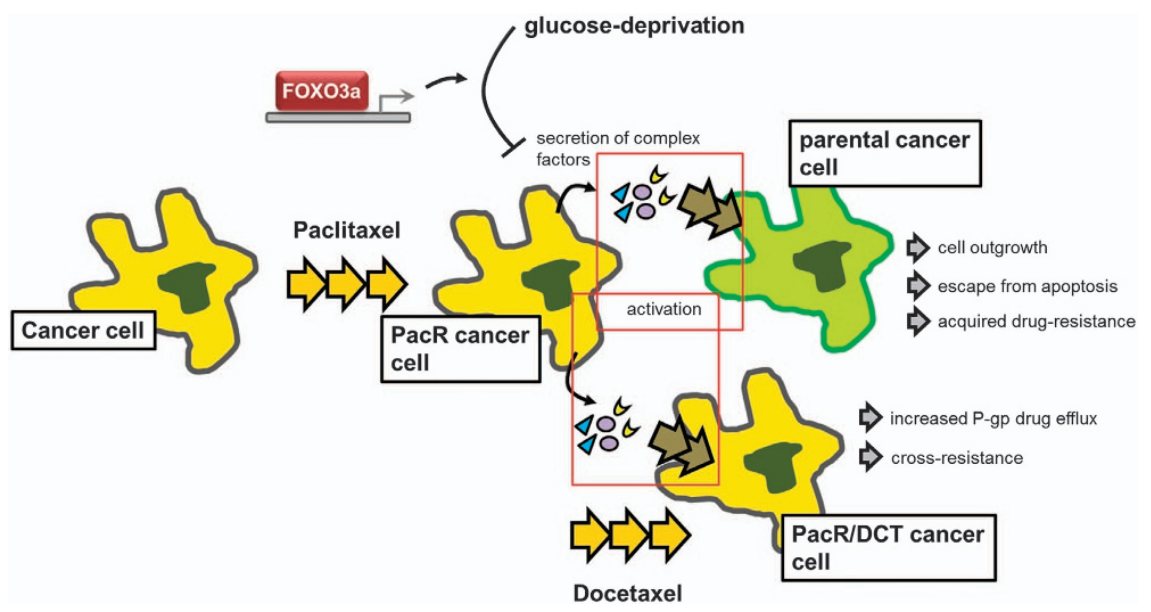

Figure 7 Proposed scheme of the effects of PacR cancer cell-derived secretomes on drug-sensitive cancer cells and regulation via glucose modification and FOXO3a. P-gp, P-glycoprotein.

of the cell growth response to PacR cell secretomes with modified glucose content (that is, enriched with $25 \mathrm{~mm}$ glucose or deprived), we found significant changes in the growth rates ( $48 \mathrm{~h}$ cell viability normalized to $24 \mathrm{~h}$ ) of parental, PacR and PacR/DCT variants in response to PacR secretomes and subsequent FOXO3a siRNA experiments (Table 2). This result suggested that targeting glycolysis in PacR cells prevents the secretion of factors that promote cross-resistance in drugresistant cancer cells, which effectively addresses how multiplicity in drug resistance is primarily acquired in drug-resistant cancer cells. To support this claim, we analyzed the protein expression levels of $\mathrm{P}$-gp and FOXO3a/Akt signaling in PacR/DCT cells after exposure to either control (normal glucose) or glucose-deprived PacR cell secretomes. Deprivation of glucose in PacR cell secretomes downregulated the expression levels of P-gp, phospho-Akt, phospho-FOXO3a and IкB $\alpha$, with minimal changes in p27Kip1. These effects were augmented by the suppression of FOXO3a transcription (Figure 6c). AMP-activated protein kinase (AMPK) has been implicated in the regulation of glycolysis in several cancer types. ${ }^{54}$ More specifically, the phosphorylation of AMPK- $\alpha$ is involved in glucose-dependent Akt activity, possibly through the suppression of AMPK and the activation of Akt by glucose, ${ }^{55}$ affecting ABCG2 expression; ${ }^{48}$ ABCG2 is a member of the ATP-binding cassette family known to mediate drug efflux pumps. We found differential expression of drug-induced phospho-AMPK- $\alpha$ in A549-PacR/DCT and PC-3-PacR/DCT cells after transient transfection with FOXO3a siRNA under glucose deprivation; the expression was inhibited in A549-PacR/DCT cells but upregulated in PC-3-PacR/ DCT cells. This differential expression might be associated with the wild-type status of LKB1 in the parental A549 $(\mathrm{LKB} 1-/-)$ and PC-3 (LKB1+/+) cells, which differentially exhibit homozygous inactivating mutations of endogenous LKB1, a regulator of AMPK activation and phosphorylation. ${ }^{56,57}$ Nevertheless, docetaxel induced phospho-AMPK- $\alpha$ activation in both A549-PacR/DCT and PC-3-PacR/DCT cells after silencing of FOXO3a and cell exposure to glucose-deprived
PacR cell secretomes (Figure 6d). Moreover, we found enhancement of the docetaxel-induced depletion of ATP by silencing FOXO3a in A549-PacR/DCT cells after exposure to both control and glucose-deprived PacR cell secretomes (Figure 6e). These results are consistent with those of previous reports that shown defective ATP production upon glucose content modification in drug-resistant cancer cells..$^{58,59}$

\section{DISCUSSION}

Paclitaxel-based therapies, alone or in combination with kinase inhibitors such as erlotinib, carboplatin and flavopiridol, have shown significant clinical efficacy in patients with advanced non-small cell lung cancer or prostate cancer. ${ }^{60-62}$ Although adverse events are sometimes observed, they do not occur as commonly as they do when paclitaxel is used alone. Nonetheless, taxane-based therapies have been shown to have acceptable tolerability and have led to improvements in the duration and quality of life for most of these patients. ${ }^{63,64}$ However, drug resistance inevitably develops with progressive disease, representing a major obstacle for improving overall survival. Paclitaxel-resistant cancer types have been reported to develop more rapid cross-resistance to a variety of unrelated drugs. ${ }^{6-67}$ However, the development of rapid multiplicity and reaction to changes in the microenvironment during tumor progression for these paclitaxel-resistant tumor populations in acquired cross-resistance are not yet well understood.

Previously, we reported that microtubule stability and ABCB1 could be regulated by FOXO3a to induce multiplicity in acquired cross-resistance to unrelated drugs such as 5-fluorouracil and cisplatin in paclitaxel-resistant lung and prostate cancer cells. This function may augment the release of drug resistance-promoting secreted factors. ${ }^{31}$ In a more physiologically relevant context, this function of FOXO3a has been well described with the specific role of therapy-induced cancer cell secretomes (in the form of $\mathrm{CM}$ ) in promoting resistance and progression in cancer cells exposed to these secretomes both in vitro and in vivo. ${ }^{32,53} \mathrm{~A}$ better 
understanding of how targeted glycolysis mediates this process could lead to the improved efficacy of current targeted therapies.

To model how secretomes derived from cancer cells with high-fold resistance to paclitaxel affect drug-sensitive cells, we employed the established PacR cancer cell lines (A549-PacR and $\mathrm{PC}-3-\mathrm{PacR}$ ), which were developed over the course of $>12$ months and were 200 times more resistant to paclitaxel. We collected the CM of these PacR cells for $48 \mathrm{~h}$ and cultured the drug-sensitive A549 and PC-3 cells with these cell secretomes under normal culture conditions. We found that the PacR cell-derived secretomes significantly contribute to cell growth and consistently limit the efficacy of drugs, such as paclitaxel, docetaxel and cisplatin. Mechanistic evaluation clearly demonstrates that FOXO3a/Akt signaling triggers the ability of these PacR cell secretomes to mediate their effects on drug-sensitive cells, indicating a tight link between FOXO3a and acquired drug resistance. This finding was further extended to glucose modification in PacR cells. We found that GD prevents the secretion of complex factors by PacR cells that promote cell proliferation, escape from apoptosis and drug resistance in drug-sensitive cancer cells. Consistent with these findings, the enrichment of glucose in PacR cells either maintained or enhanced the cell growth-promoting effects of the PacR cell secretomes, providing complementary evidence that targeting glycolysis in PacR cancer cells mediates the secretion of drug resistance-promoting factors.

FOXO3a has been observed to amplify PI3K/Akt signaling in drug-resistant cancer cells, which are then characterized with elevated FOXO3a expression and activity. ${ }^{15}$ FOXO3a is recruited to the $\mathrm{ABCB} 1$ promoter regulating $\mathrm{ABCB} 1$ expression in response to chemotherapy drugs. ${ }^{14}$ Indeed, we found that the suppression of FOXO3a inhibited $\mathrm{P}$-gp/ABCB1 expression in PacR cancer cells exposed to PacR cell secretomes, which underwent either glucose enrichment or GD. Under both circumstances, FOXO3a mediated the effects of the secretomes on docetaxel drug sensitivity and escape from apoptosis, as demonstrated by the regulated expression of cell death markers and TRAIL-induced regulation. FOXO3a-driven glycolysis has been closely associated with downstream signals of Akt through tuberous sclerosis complex 1 (TSC1). ${ }^{44}$ Hyperactivation of PI3K/Akt activity has been described as a consequence of chronic induction of $\mathrm{FOXO} 3 \mathrm{a}$ in multidrug-resistant leukemic cells. ${ }^{15}$ However, the exact mechanisms by which FOXO3a might be able to direct Akt through TSC1 to regulate the response of drug-sensitive cancer cells to PacR secretomes remain unclear and must be further investigated.

To further determine whether the promotion of docetaxel cross-resistance in PacR cells is evident, we employed the established PacR/DCT cells, which were developed to have transient cross-resistance to docetaxel and a retained high-fold resistance to paclitaxel. By depriving PacR cells of glucose, the secreted factors failed to augment docetaxel resistance, which was amplified by silencing FOXO3a. These findings highlight an element of complexity associated with the mechanisms that drive the development of cross-resistance in PacR cancer cells; nevertheless, these findings further confirm that drug-resistant cancer cell secretomes promote resistance and outgrowth in drug-sensitive cells and confirm that targeted glycolysis can amend the process.

Further studies were performed to reveal these secreted factors of PacR-derived cell secretomes that cause the permissive stimulation of outgrowth and support the survival of drug-sensitive cancer cells. The limited efficacy of some drugs has been attributed to specific cytokines that support cell growth and the acquisition of aggressive phenotypes that promote the survival of drug-sensitive cancer cells. We found that the drug-stimulated levels of IFN- $\alpha$, IFN- $\lambda$ and TNF- $\alpha$ were alleviated in cancer cells by treatment with PacR cell secretomes, suggesting that PacR cell secretomes stimulate cell growth and resistance in drug-sensitive cancer cells (Figure 7).

In summary, the present findings reveal that PacR cancer cells secrete tumor growth-promoting factors that stimulate regulatory signals to shift apoptosis and develop acquired drug resistance in drug-sensitive cancer cells and cross-resistance in PacR cancer cells. It is especially notable that these findings support, at the basic level, how we can further regulate the effects of therapy-induced secretomes by targeting glycolysis, which appeared to be mediated by FOXO3a signaling. Considering that paclitaxel-based treatments are carefully being clinically evaluated, these findings reveal an approach to strategically improve the efficacy of therapies involving paclitaxel for successfully overcoming acquired drug resistance.

\section{CONFLICT OF INTEREST}

The authors declare no conflict of interest.

\section{ACKNOWLEDGEMENTS}

We thank Jayoung Song for the development and culture maintenance of the PC-3 and PC-3-PacR cell lines. This work was supported by a grant from procurement and development of biological resources funded by the Ministry of Education Science and Technology of the Korean government (2011-00499) and a National Research Foundation of Korea (NRF) grant funded by the Korean Government (MEST; no. 2009-0083533).

1 Garnett MJ, Edelman EJ, Heidorn SJ, Greenman CD, Dastur A, Lau KW et al. Systematic identification of genomic markers of drug sensitivity in cancer cells. Nature 2012; 483: 570-575.

2 Cancer Cell Line Encyclopedia Consortium and The Genomics of Drug Sensitivity in Cancer Consortium, Pharmacogenomic agreement between two cancer cell line data sets. Nature 2015; 528: 84-87.

3 Niederst MJ, Engelman JA. Bypass mechanisms of resistance to receptor tyrosine kinase inhibition in lung cancer. Sci Signal 2013; 6: re6.

4 Zasadil LM, Andersen KA, Yeum D, Rocque GB, Wilke LG, Tevaarwerk AJ et al. Cytotoxicity of paclitaxel in breast cancer is due to chromosome missegragation on multipolar spindles. Sci Trans/ Med 2014; 6: $229 \mathrm{ra} 43$.

5 Jin MS, Oldham ML, Zhang Q, Chen J. Crystal structure of the multidrug transporter P-glycoprotein from Caenorhabditis elegans. Nature 2012; 490: 566-569.

6 Gottesman MM, Fojo T, Bates SE. Multidrug resistance in cancer: role of ATP-dependent transpoters. Nat Rev Cancer 2002; 2: 48-58.

7 Goncalves A, Braguer D, Kamath K, Martello L, Briand C, Horwitz S et al. Resistance to taxol in lung cancer cells associated with increased microtubule dynamics. Proc Natl Acad USA 2001; 98: 11737-11742. 
8 Binkhathlan Z, Lavasanifar A. P-glycoprotein inhibition as a therapeutic approach for overcoming multidrug resistance in cancer: current status and future perspectives. Curr Cancer Drug Targets 2012; 13: 326-346.

9 Pelicano H, Martin DS, Xu RH, Huang P. Glycolysis inhibition for anticancer treatment. Oncogene 2006; 25: 4633-4646.

10 Ganapathy-Kanniappan S, Geschwind J-F. Tumor glycolysis as a target for cancer therapy: progress and prospects. Mol Cancer 2013; 12: 152.

11 Nakano A, Takashima S. LKB1 and AMP-activated protein kinase: regulators of cell polarity. Genes Cells 2012; 17: 737-747.

12 Lee AS. GRP78 induction in cancer: therapeutic and prognostic implications. Cancer Res 2007; 67: 3496-3499.

13 Hanahan D, Weinberg RA. Hallmarks of cancer: the next generation. Cell 2011; 144: 646-674.

14 Hui RC-Y, Francis RE, Guest SK, Costa JR, Gomes AR, Myatt SS et al. Doxorubicin activates $\mathrm{FOXO3a}$ to induce the expression of multidrug resistance gene ABCB1 (MDR1) in K562 leukemic cells. Mol Cancer Ther 2008; 7: 670-678.

15 Hui RC-Y Gomes AR, Constantinidou D, Costa JR, Karadedou CT, Fernandez de Mattos $\mathrm{S}$ et al. The forkhead transcription factor FOXO3a increases phosphoinositide-3 kinase/Akt activity in drug-resistant leukemic cell through induction of PIK3CA expression. Mol Cell Biol 2008; 28: 5886-5898.

16 Zhao F, Lam EW. Role of the forkhead transcription factor FOXO-FOXM1 axis in cancer and drug resistance. Front Med 2012; 6: 376-380.

17 Greer EL, Brunet A. FOXO transcription factors at the interface between longevity and tumor suppression. Oncogene 2005; 24: 7410-7425.

18 Wilson TR, Johnston PG, Longley DB. Anti-apoptotic mechanisms of drug resistance in cancer. Curr Cancer Drug Targets 2009; 9: 307-319.

19 Bae SY, Hong JY, Lee HJ, Park HJ, Lee SK. Targeting the degradation of AXL receptor tyrosine kinase to overcome resistance in gefitinib-resistant non-small cell lung cancer. Oncotarget 2015; 6: 10146-10160.

20 Aldonza MB, Hong JY, Bae SY, Song J, Kim WK, Oh J et al. Suppression of MAPK signaling and reversal of mTOR-dependent MDR1-associated multidrug resistance by $21 \alpha$-methylmelianodiol in lung cancer cells. PLOS ONE 2015; 10: e0127841.

21 Kim EH, Min HY, Chung HJ, Song J, Park HJ, Kim S et al. Anti-proliferative activity and suppression of P-glycoprotein by (-)-antofine, a natural phenanthroindolizidine alkaloid, in paclitaxel-resistant human lung cancer cells. Food Chem Toxicol 2012; 50: 1060-1065.

22 Luo H, Yang Y, Duan J, Wu P, Jiang Q, Xu C. PTEN-regulated AKT/FoxO3a/ Bim signaling contributes to reactive oxygen species-mediated apoptosis in selenite-treated colorectal cancer cells. Cell Death Dis 2013; 4: e481.

23 Shastri MD, Stewart N, Horne J, Peterson GM, Gueven N, Sohal SS et al. In-vitro suppression of IL-6 and IL-8 release from human pulmonary epithelial cells by non-anticoagulant fraction of enoxaparin. PLOS ONE 2012; 10: e0126763.

24 Darzynkiewicz Z, Galkowski D, Zhao H. Analysis of apoptosis by cytometry using TUNEL assay. Methods 2008; 44: 250-254.

25 McManus KJ, Barrett IJ, Nouhi Y, Hieter P. Specific synthetic lethal killing of RAD54B-deficient human colorectal cancer cells by FEN1 silencing. Proc Natl Acad Sci USA 2009; 106: 3276-3281.

26 Pozarowski P, Darzynkiewicz Z. Analysis of cell cycle by flow cytometry. Methods Mol Biol 2004; 281: 301-311.

27 Horke S, Witte I, Wilgenbus P, Kruger M, Strand D, Forstermann U. Paraoxonase-2 reduces oxidative stress in vascular cells and decreases endoplasmic reticulum stress-induced caspase activation. Circulation 2007; 115: 2055-2064.

$28 \mathrm{Na}$ SS, Aldonza MB, Sung HJ, Kim YI, Son YS, Cho S et al. Stanniocalcin-2 (STC2): a potential lung cancer biomarker promotes lung cancer metastasis and progression. Biochim Biophys Acta 2015; 1854: 668-676.

29 Ahn JM, Sung HJ, Yoon YH, Kim BG, Yang WS, Lee C et al. Integrated glycoproteomics demonstrates fucosylated serum paraoxonase 1 alterations in small cell lung cancer. Mol Cell Proteomics 2014; 13: 30-48.

30 Junttila MR, de Sauvage FJ. Influence of tumour micro-environment heterogeneity on therapeutic response. Nature 2013; 501: 346-354.

31 Aldonza MBD, Hong JY, Alinsug MV, Song J, Lee SK. Multiplicity of acquired cross-resistance in paclitaxel-resistant cancer cells is associated with feedback control of TUBB3 via FOXO3a-mediated ABCB1 regulation. Oncotarget 2016; 7: 34395-34419.

32 Sun Y, Campisi J, Higano C, Beer TM, Porter P, Coleman I et al. Treatment-induced damage to the tumor microenvironment promotes prostate cancer therapy resistance through WNT16B. Nat Med 2012; 18: $1359-1368$.
33 Lee HJ, Zhuang G, Cao Y, Du P, Kim HJ, Settleman J. Drug resistance via feedback activation of Stat3 in oncogene-addicted cancer cells. Cancer Cell 2014; 26: 207-221.

34 Kim JJ, Yin B, Christudass CS, Terada N, Rajagopalan K, Fabry B et al. Acquisition of paclitaxel resistance is associated with a more aggressive and invasive phenotype in prostate cancer. J Cell Biochem 2013; 114: 1286-1293.

35 Bateman NW, Jaworski E, Ao W, Wang G, Litzi T, Dubil E et al. Elevated AKAP12 in paclitaxel-resistant serous ovarian cancer cells is prognostic and predictive of poor survival in patients. J Proteome Res 2015; 14: $1900-1910$

36 Aramburu J, Ortells MC, Tejedor S, Buxade M, Lopez-Rodriguez C. Transcriptional regulation of the stress response by mTOR. Sci Signal 2014; 7: re2.

37 Pyndiah S, Tanida S, Ahmed KM, Cassimere EK, Choe C, Sakamuro D. C-MYC suppresses BIN1 to release poly(ADP-ribose) polymerase 1: a mechanism by which cancer cells acquire cisplatin resistance. Sci Signal 2011; 4: ra19.

38 Allen JE, Krigsfeld G, Mayes PA, Patel L, Dicker DT, Patel AS et al. Dual inactivation of Akt and ERK by TIC10 signals Foxo3a nuclear translocation, TRAIL gene induction, and potent antitumor effects. Sci Trans/ Med 2013; 5: 171 ra17.

39 Gomes AR, Brosens JJ, Lam EW-F. Resist or die: FOXO transcription factors determine the cellular response to chemotherapy. Cell Cycle 2008; 7: 3133-3135.

40 Hill R, Kalathur RKR, Callejas S, Colaco L, Brandao R, Serelde B et al. A novel phosphatidylinositol 3-kinase (PI3K) inhibitor directs a potent FOXO-dependent, p53-independent cell cycle arrest phenotype characterized by the differential induction of a subset of FOXO-regulated genes. Breast Cancer Res 2014; 16: 482.

41 Santo EE, Stroeken P, Sluis PV, Koster J, Versteeg R, Westerhout EM. FOXO3a is a major target of inactivation by PI3K/AKT signaling in aggressive neuroblastoma. Cancer Res 2013; 73: 2189-2198.

42 Lyon RC, Cohen JS, Faustino PJ, Megnin F, Myers CE. Glucose-metabolism in drug-sensitive and drug-resistant human breast cancer cells monitored by magnetic resonance spectroscopy. Cancer Res 1988; 48: 870-877.

43 Riganti C, Gazzano E, Polimeni M, Aldieri E, Ghigo D. The pentose phosphate pathway: an antioxidant defense and a crossroad in tumor cell fate. Free Radic Biol Med 2012; 53: 421-436.

44 Khatri S, Yepiskoposyan H, Gallo CA, Tandon P, Plas DR. FOXO3a regulates glycolysis via transcriptional control of tumor suppressor TSC1. J Biol Chem 2010; 285: 15960-15965.

45 Moley KH, Mueckler MM. Glucose transport and apoptosis. Apoptosis 2000: 5: 99-105.

46 Fong MY, Zhou W, Liu L, Alontaga AY, Chandra M, Ashby J et al. Breast-cancer-secreted miR-122 reprograms glucose metabolism in premetastatic niche to promote metastasis. Nat Cell Biol 2015; 17: 183-194.

47 Zhao Y, Butler EB, Tan M. Targeting cellular metabolism to improve cancer therapeutics. Cell Death Dis 2013; 4: e532.

48 Schulze A, Harris AL. How cancer metabolism is tuned for proliferation and vulnerable to disruption. Nature 2012; 491: 364-373.

49 Liu PP, Liao J, Tang ZJ, Wu WJ, Yang J, Zeng ZL et al. Metabolic regulation of cancer cell side population by glucose through activation of the Akt pathway. Cell Death Differ 2014; 21: 124-135.

50 Nicoletti I, Migliorati G, Pagliacci MC, Grignani F, Riccardi C. A rapid and simple method for measuring thymocyte apoptosis by propium iodide staining and cytometry. J Immunol Methods 1991; 139: 271-279.

51 Fraker PJ, King LE, Lill-Elghanian D, Telford WG. Quantification of apoptotic events in pure and heterogenous populations of cells using the flow cytometer. Methods Cell Biol 1995; 46: 57-76.

52 Gottesman MM, Lavi O, Hall MD, Gillet JP. Toward a better understanding of the complexity of cancer drug resistance. Annu Rev Pharmacol Toxicol 2016; 56: 85-102.

53 Komurov K, Tseng JT, Muller M, Seviour EG, Moss TG, Yang L et al. The glucose-deprivation network counteracts lapatinib-induced toxicity in resistant ErbB2-positive breast cancer cells. Mol Syst Biol 2012; 8: 596.

54 Obenauf AC, Zou Y, Ji AL, Vanharanta S, Shu W, Shi H et al. Therapy-induced tumour secretomes promote resistance and tumour progression. Nature 2015; 520: 368-372.

55 Faubert B, Boily G, Izreig S, Griss T, Samborska B, Dong Z et al. AMPK is a negative regulator of the Warburg effect and suppresses tumor growth in vivo. Cell Metab 2013; 17: 113-124. 
56 King TD, Song L, Jope RS. AMP-activated kinase (AMPK) activating agents cause dephosphorylation of Akt and glycogen synthase-kinase-3. Biochem Pharmacol 2006; 71: 1637-1647.

57 Shackelford DB, Shaw RJ. The LKB1-AMPK pathway: metabolism and growth control in tumour suppression. Nat Rev Cancer 2009; 9: 563-575.

58 Kottakis F, Bardeesy N. LKB1-AMPK axis revisited. Cell Res 2012; 22. 1617-1620.

59 Zhou Y, Tozzi F, Chen J, Fan F, Xia L, Wang J et al. Intracellular ATP levels are a pivotal determinant of chemoresistance in colon cancer cells. Cancer Res 2012; 72: 304-314.

60 Maschek G, Savaraj N, Priebe W, Braunschweiger P, Hamilton K, Tidmarsh GF et al. 2-deoxy-D-glucose increases the efficacy of adriamycin and paclitaxel in human osteosarcoma and non-small cell lung cancers in vivo. Cancer Res 2004; 64: 31-34.

61 Patnaik A, Wood D, Tolcher AW, Hamilton M, Kreisberg JI, Hammond LA et al. Phase I, pharmacokinetic, and biological study of erlotinib in combination with paclitaxel and carboplatin in patients with advanced solid tumors. Clin Cancer Res 2006; 12: 7406-7413.

62 Chen YM, Perng RP, Tsai CM, Whang-Peng J. A phase II randomized study of paclitaxel plus carboplatin or cisplatin against chemo-naïve inoperable non-small cell lung cancer in the elderly. J Thorac Oncol 2006; 1: 141-145.

63 Schwartz GK, O'Reilly E, Ilson D, Saltz L, Sharma S, Tong W et al. Phase I study of the cyclin-dependent kinase inhibitor flavopiridol in combination with paclitaxel in patients with advanced solid tumors. J Clin Oncol 2002; 20: 2157-2170.
64 Ramalingam SS, Maitland ML, Frankel P, Argiris AE, Koczywas M, Gitlitz B et al. Carboplatin and paclitaxel in combination with either vorinostat or placebo for first-line therapy of advanced non-small-cell lung cancer. J Clin Oncol 2010; 28: 56-62.

65 Tuma RS. Taxol's journey from discovery to use: lessons and updates. Oncol Times 2003; 25: 52-57.

66 Weaver BA. How taxol/paclitaxel kills cancer cells. Mol Biol Cell 2014; 25 : 2677-2681.

67 McDermott M, Eustace AJ, Busschots S, Breen L, Crown J, Clynes M et al. In vitro development of chemotherapy and targeted therapy drug-resistant cancer cell lines: a practical guide with case studies. Front Oncol 2014; 4: 40 .

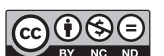

This work is licensed under a Creative Commons Attribution-NonCommercial-NoDerivs 4.0 International License. The images or other third party material in this article are included in the article's Creative Commons license, unless indicated otherwise in the credit line; if the material is not included under the Creative Commons license, users will need to obtain permission from the license holder to reproduce the material. To view a copy of this license, visit http://creativecommons.org/licenses/by-nc-nd/4.0/

Supplementary Information accompanies the paper on Experimental \& Molecular Medicine website (http://www.nature.com/emm) 